Supplementary information $5 \mathrm{a}$ - Country details on sub-national statistics for cattle (year and level of observation) by continental tile.

\title{
Africa Tile
}

\begin{tabular}{|c|c|c|c|c|}
\hline COUNTRY & YEAR & LEVEL & $\begin{array}{l}\text { Totals in } \\
\text { GLIMS }\end{array}$ & SOURCE \\
\hline Algeria & 2002 & 1 & $1,345,593$ & $\begin{array}{l}\text { Direction des Services Vétérinaires - Ministère de l'Agriculture et du Développement Rural } 2003 \text { (GLIMS data } \\
\text { warehouse) }\end{array}$ \\
\hline Angola & 2011 & 1 & $4,586,569$ & $\begin{array}{l}\text { CountrySTAT - Food and agriculture data network: Número de animais vivos by Ano, Especie, Nivel } \\
\text { administrativo. Online data: http:// countrystat.org/home.aspx?c=AGO\&ta=007SPD035\&tr=21 }\end{array}$ \\
\hline Benin & 2011 & 2 & $2,058,000$ & $\begin{array}{l}\text { CountrySTAT - Food and agriculture data network: Élevage - Total des effectifs d'animaux vivants } 2010-2012 . \\
\text { Online data: } \text { http://www.countrystat.org/home.aspx?c=BEN\&ta=053SPD135\&tr=21 }\end{array}$ \\
\hline Botswana & 2010 & 2 & $2,249,364$ & Central Statistics Office of Botswana: 2009 and 2010 Annual Agricultural Surveys (GLIMS data warehouse) \\
\hline Burkina Faso & 2009 & 2 & $8,233,900$ & $\begin{array}{l}\text { Ministère de l'économie et de développement, Direction General des Prévisions et des Statistiques Agricoles: Les } \\
\text { régions en chiffres, } 2008 \text { \& } 2010 \text { (GLIMS data warehouse) }\end{array}$ \\
\hline Burundi & 2005 & 2 & 364,732 & $\begin{array}{l}\text { Direction Provinciale de l'Agriculture et de l'Élevage, Burundi (DPEA): Monographies des les Communes } 2006 \\
\text { (GLIMS data warehouse) }\end{array}$ \\
\hline Cameroon & 2007 & 1 & $1,253,931$ & CountrySTAT - Food and agriculture data network (GLIMS data warehouse) \\
\hline Cape Verde & 2004 & 2 & 22,306 & Directorate of livestock, Cape Verde: Recenseamento Geral da Agricultura, 2004 (GLIMS data warehouse) \\
\hline $\begin{array}{l}\text { Central African } \\
\text { Republic }\end{array}$ & 1990 & 1 & $2,501,269$ & $\begin{array}{l}\text { Environmental Research Group Oxford, ERGO: FAO, 2004. Agro-ecological distributions for Africa (Data CD) } \\
\text { (GLIMS data warehouse) }\end{array}$ \\
\hline Chad & 2007 & 1 & $7,688,188$ & $\begin{array}{l}\text { Direction de l'Élevage et des Ressources Animales: Effectifs du cheptel national par région administrative } 2007 \\
\text { (GLIMS data warehouse) }\end{array}$ \\
\hline Comoros & 2004 & 1 & 37,200 & $\begin{array}{l}\text { FAO animal production and health division: Rapport National Sur l'Etat des Ressources Génétiques Animales } \\
\text { (GLIMS data warehouse) }\end{array}$ \\
\hline Congo & 1996 & 1 & 72,100 & $\begin{array}{l}\text { Centre National de la Statistique et des Études Économiques, Congo: Estimations des comptes nationaux de } \\
\text { 1997-2000 (GLIMS data warehouse) }\end{array}$ \\
\hline Côte d'Ivoire & 2001 & 1 & $1,135,196$ & $\begin{array}{l}\text { Ministère de l'Agriculture et des Ressources Animales, Direction de la Programmation, Côte d'Ivoire: } \\
\text { Recensement National de l'Agriculture } 2001 \text { (GLIMS data warehouse) }\end{array}$ \\
\hline $\begin{array}{l}\text { Democratic } \\
\text { Republic of the } \\
\text { Congo }\end{array}$ & 2004 & 1 & 2,899 & $\begin{array}{l}\text { Centre for Ticks and Tick-Borne Diseases: Preliminary census data from SADC and Eastern Africa (GLIMS data } \\
\text { warehouse) }\end{array}$ \\
\hline Djibouti & 1998 & 1 & 297,001 & Ministry of Agriculture of Djibouti through IGAD secretariat staff, data for 1998 (GLIMS data warehouse) \\
\hline Egypt & 2011 & 1 & $4,779,743$ & $\begin{array}{l}\text { Central Agency for Public Mobilization and Statistics, Egypt: Estimated numbers of livestock and animals by } \\
\text { Governorate } 2011 \text { (GLIMS data warehouse) }\end{array}$ \\
\hline
\end{tabular}


Eritrea

Ethiopia

Gabon

Gambia

Ghana

1996

Guinea

Guinea-Bissau$$
200
$$

Kenya

Lesotho

Liberia

Madagascar

Malawi

Mali

Mauritania

Mauritius

Mayotte

Morocco

Mozambique

Namibia

Niger

Nigeria
320 International Programme for the Development of the Meat Sector and International Programme for Coordinating the Development of the Dairy Sector. Republic of Equatorial Guinea, Draft Report, FAO, Rome, Italy.

1,927,137 Environmental Research Group Oxford, ERGO: GIEWS special reports. Online data: http://www.fao.org/docrep/005/y7678e/y7678e00.htm

45,758,978 Central Statistical Authority of Ethiopia: Agricultural Census 2001-2002 (GLIMS data warehouse)

18,321 Environmental Research Group Oxford, ERGO: Agro-ecological distributions for Africa (GLIMS data warehouse)

323,167 Agricultural Statistics and Resources Economics Unit (ASRE), Government of the Gambia: Report of the Agricultural Census of The Gambia 2001/2002 Volume 1 and Volume 2 (GLIMS data warehouse)

1,247,861 Veterinary Services of Ghana: Livestock Census, 1994-1996 (GLIMS data warehouse)

5,234,783 Réseau des Systèmes d'Information des Marchés en Afrique de l'Ouest: Stats cheptel. Online data: http://www.resimao.org/html/fr/Guinee/stats\#cheptel

1,121,555 Ministère de l'Agriculture et du Développement Rural, Guinée-Bissau: Synthèse des résultats du Recensement National de l' Elevage en Guinée-Bissau, 2009 (GLIMS data warehouse)

10,714,381 Ministry of Livestock Development, Kenya: Compilation of divisional data 2005 (GLIMS data warehouse)

626,344 Bureau of Statistics of Lesotho: 2009/10 Lesotho Agricultural Census, Livestock Report (GLIMS data warehouse)

12,598 Ministry of Agriculture Regional Livestock Officers, Liberia: Estimates On Livestock Population in Liberia (GLIMS data warehouse)

9,500,138 Ministère de l'Agriculture, Secrétariat General, Direction de la Prévision et des Etudes Economiques, Madagascar: Recensement de l'agriculture (RA) - Campagne agricole 2004-2005 (GLIMS data warehouse)

884,132 Agriculture Statistics Division of the National Statistical Office of Malawi (NSO): National Census of Agriculture and Livestock (October 2006 - October 2007) (GLIMS data warehouse)

9,438,181 CountrySTAT - Food and agriculture data network: Élevage - Total des effectifs d'animaux vivants. Online data: http://www.countrystat.org/home.aspx?c=MLI\&tr=21

795,500 Office of National Statistics: Monographies Des Régions (GLIMS data warehouse)

5,365 Central Statistic Office of Mauritius: Digest of Agricultural Statistics 2011 (GLIMS data warehouse)

17,150 Agreste la Statistique Agricole, Le Ministère de l'Agriculture, de l'Alimentation, de la Pêche et de la Ruralité, France: Recensement Agricole 2010. Online data: http://agriculture.gouv.fr/recensement-agricole-2010

2,651,200 Ministry of Agriculture, Rural Development and Sea Fisheries: Maroc Régions 2008 (GLIMS data warehouse)

590,363 Direccao Nacional De Pecuaria, Mozambique: Annual Reports, 2003 (GLIMS data warehouse)

2,388,780 FAO Special Report, FAO/WFP Crop, Livestock and Food Security Assessment Mission to Namibia (GLIMS data warehouse)

7,336,087 Ministère des Ressources Animales, Niger: Recensement Général de l'Agriculture et du Cheptel. Volume II. Résultats Définitifs (Volet cheptel). Juin 2007. (Projet GCP/NER/041/EC). (GLIMS data warehouse)

25,019,700 National Bureau of Statistics, Nigeria: Agricultural Census 2007 (GLIMS data warehouse) 


\begin{tabular}{|c|c|c|c|c|}
\hline Rwanda & 2008 & 2 & $1,548,970$ & $\begin{array}{l}\text { National Institute of Statistics of Rwanda: National Agricultural Survey } 2008 \text { (Final report) (GLIMS data } \\
\text { warehouse) }\end{array}$ \\
\hline Réunion & 2010 & 2 & 16,316 & $\begin{array}{l}\text { Agreste la Statistique Agricole, Le Ministère de l'Agriculture, de l'Alimentation, de la Pêche et de la Ruralité, } \\
\text { France: Recensement Agricole 2010. Online data: http://agriculture.gouv.fr//recensement-agricole-2010 }\end{array}$ \\
\hline Saint Helena & 2009 & 1 & 598 & Agriculture and Natural Resources Department of St. Helena: Livestock Census 2009 (GLIMS data warehouse) \\
\hline Senegal & 2009 & 2 & $3,260,867$ & $\begin{array}{l}\text { Agence Nationale de la Statistique et de la Démographie du Sénégal: Live Data Base (LDB). Online data: } \\
\text { http://www.ansd.sn/publications_SES_region.html }\end{array}$ \\
\hline Sierra Leone & 2004 & 2 & 98,632 & $\begin{array}{l}\text { Ministry of Agriculture \& Food Security of Sierra Leone: } 2004 \text { Populations and Housing census Statistical Office } \\
\text { (GLIMS data warehouse) }\end{array}$ \\
\hline Somalia & 1996 & 6 & $4,409,430$ & $\begin{array}{l}\text { Food Security Assessment Unit (FSAU) in 1999: Livestock Populations in Various Zones of Somalia (FSAU } \\
\text { data) (GLIMS data warehouse) }\end{array}$ \\
\hline South Africa & 2011 & 1 & $13,871,000$ & $\begin{array}{l}\text { Ministry of Agriculture, Forestry and Fisheries of South Africa: Trends in the Agricultural Sector, } 2011 \text { (GLIMS } \\
\text { data warehouse) }\end{array}$ \\
\hline Sudan & 2009 & 1 & $41,563,002$ & $\begin{array}{l}\text { Ministry of Animal Resources and fisheries, Sudan: Statistical Year Book for the Year } 2009 \text { (GLIMS data } \\
\text { warehouse) }\end{array}$ \\
\hline Swaziland & 2010 & 1 & 619,753 & $\begin{array}{l}\text { Directorate of Veterinary and Livestock Services: Swaziland Livestock Statistics, Census } 2010 \text { (GLIMS data } \\
\text { warehouse) }\end{array}$ \\
\hline Togo & 1995 & 1 & 201,078 & Data from Dr. Guy Hendrickx: Round Survey, 1995 (GLIMS data warehouse) \\
\hline Tunisia & 2010 & 1 & 670,980 & $\begin{array}{l}\text { Ministère de l'Agriculture de l'Environnement et des Ressources Hydrauliques, Tunisia: Annuaire Statistique } \\
2010 \text { (GLIMS data warehouse) }\end{array}$ \\
\hline Uganda & 2008 & 4 & $10,016,659$ & Uganda Bureau of Statistics: Uganda census 2008 - Data acquired from Dr. W.Wint (GLIMS data warehouse) \\
\hline $\begin{array}{l}\text { United Republic of } \\
\text { Tanzania }\end{array}$ & 2009 & 2 & $18,713,654$ & CountrySTAT - Food and agriculture data network (GLIMS data warehouse) \\
\hline Western Sahara & 2009 & 1 & 1,300 & Direction de la Statistique du Maroc: Annuaires Statistiques des Régions (GLIMS data warehouse) \\
\hline Zambia & 2009 & 1 & $3,445,630$ & $\begin{array}{l}\text { Department of Research and Specialist Services, Zambia: National Livestock Census by Province } 1994 \text { to } 2009 \\
\text { (GLIMS data warehouse) }\end{array}$ \\
\hline Zimbabwe & 2008 & 2 & $5,106,673$ & Department of Livestock and Veterinary Services: Livestock Census Summary (GLIMS data warehouse) \\
\hline
\end{tabular}




\section{Asia Tile}

\begin{tabular}{|c|c|c|c|c|}
\hline COUNTRY & YEAR & LEVEL & Totals in GLIMS & SOURCE \\
\hline Afghanistan & 2003 & 2 & $3,706,186$ & $\begin{array}{l}\text { Ministry of Agriculture, Irrigation and Livestock of Afghanistan: Afghanistan - National Livestock Census } \\
\text { 2002-2003 - Final Report. Online data: } \\
\text { http://reliefweb.int/sites/reliefweb.int/files/resources/58C374339FC582F149256DF30009D60F-faO-afg- } \\
\text { 4dec.pdf }\end{array}$ \\
\hline Armenia & 2012 & 1 & 599,243 & $\begin{array}{l}\text { National statistical service of the republic of Armenia: Livestock Census, 2012. Online data: } \\
\text { http://www.armstat.am/file/article/anas_2012.pdf }\end{array}$ \\
\hline Azerbaijan & 2011 & 1 & $2,412,305$ & $\begin{array}{l}\text { The State Statistical Committee of the Republic of Azerbaijan: The data on regions. Online data: } \\
\text { http://www.stat.gov.az/source/agriculture/indexen.php }\end{array}$ \\
\hline Bangladesh & 2008 & 3 & $28,423,749$ & $\begin{array}{l}\text { Bangladesh Bureau of Statistics: Agriculture Census Report 2008. Online data: } \\
\text { http://www.bbs.gov.bd/PageWebMenuContent.aspx?MenuKey=224 }\end{array}$ \\
\hline Belarus & 2011 & 2 & $3,930,200$ & $\begin{array}{l}\text { National Statistical Committee of the Republic of Belarus: Agriculture of the Republic of Belarus, } 2011 \\
\text { (GLIMS data warehouse) }\end{array}$ \\
\hline Bhutan & 2009 & 2 & 303,018 & $\begin{array}{l}\text { Ministry of agriculture and forest, Royal government of Bhutan: Dzongkhag livestock sector (GLIMS data } \\
\text { warehouse) }\end{array}$ \\
\hline Bulgaria & 2010 & 2 & 572,710 & $\begin{array}{l}\text { Ministry of Agriculture and Forestry - Agrostatistics Directorate, Bulgaria: Agricultural Census 2010. Online } \\
\text { data: } \\
\text { http://www.mzh.government.bg/MZH/ShortLinks/SelskaPolitika/Agrostatistics/Structure_agricultural_h } \\
\text { oldings/Results.aspx }\end{array}$ \\
\hline Cambodia & 2011 & 2 & $3,339,623$ & Ministry of Agriculture, Forestry and Fisheries: Cambodia Livestock Census 2011 (GLIMS data warehouse) \\
\hline China & 2001 & 3 & $135,686,083$ & $\begin{array}{l}\text { China Agricultural Press, 2002. For Taiwan: Statistics Office, COA, Executive Yuan, Taiwan Statistical } \\
\text { Yearbook, } 2010 \text { (GLIMS data warehouse) }\end{array}$ \\
\hline Cyprus & 2003 & 1 & 61,053 & $\begin{array}{l}\text { Statistical Service of Cyprus: Census of Agriculture, 2003. Online data: } \\
\text { http://www.mof.gov.cy/mof/cystat/statistics.nsf/All/DE20AEA98C4DD6EEC22577A50041F0B8/\$file } \\
\text { /Census_of_Agriculture-2003.pdf?OpenElement }\end{array}$ \\
\hline Georgia & 2004 & 2 & $1,157,781$ & $\begin{array}{l}\text { National Statistics Office of Georgia: First National Agricultural Census } 2004 \text { in Georgia. Online data: } \\
\text { http://geostat.ge/cms/site_images/_files/english/agriculture/census/section7.pdf }\end{array}$ \\
\hline India & 2007 & 2 & $186,515,966$ & $\begin{array}{l}\text { Department of Animal Husbandry, Dairying \& Fisheries, Ministry of Agriculture, India: Census of } \\
\text { Agriculture, } 2007 \text { (GLIMS data warehouse) }\end{array}$ \\
\hline Indonesia & 2008 & 1 & $12,714,181$ & Department Pertanian Republik Indonesia: Buku Statistik, 2009 (GLIMS data warehouse) \\
\hline Iran, Islamic & 2005 & 1 & $7,609,359$ & Statistical Centre of Iran: Iran Statistical Yearbook 1385 (2005) (GLIMS data warehouse) \\
\hline
\end{tabular}


Nepal

Pakistan
511,644 FAO, 2003. Investment Centre Division Asia and Pacific - Service emergency operation and rehabilitation division - Special emergency programme service data for the three northern governorates. Iraq - SCR 986three-year agricultural programme in the Iraqi Northern (GLIMS data warehouse)

177,194 Central Bureau of Statistics, Agriculture Division: Annual tables from publication: agriculture in Israel 20012003. Online data: http://www.cbs.gov.il/haklaut/agrical_2003/haklaut2003_e.pdf

4,230,570 Statistics Department, Minister's Secretariat, Ministry of Agriculture, Forestry and Fisheries: The 87th Statistical Yearbook of Ministry of Agriculture Forestry and Fisheries, Japan 2011-2012. Online data: http://www.maff.go.jp/e/tokei/kikaku/nenji_e/87nenji/index.html

88,215 Department of Statistics, Jordan: The Agricultural Census 2007 (GLIMS data warehouse)

5,690,000 Agency on Statistics of the Republic of Kazakhstan: Basic indicators for 2003-2012. Online data: http://www.eng.stat.kz/digital/Agriculture/Pages/default.aspx

35,705 Central Statistical Bureau, State of Kuwait: Annual Agricultural Statistics 2009-2010. Online data: http://www.csb.gov.kw/Socan_Statistic_EN.aspx?ID=42

1,357,015 National Statistic Committee of Kyrgyzstan (GLIMS data warehouse)

1,583,334 Department of Livestock and Fisheries, Laos: Lao Livestock Census Data 2011/12 in: PDR Farm Animal Distributions, 2011: Comparative Maps of Agricultural Census and Administrative Returns (GLIMS data warehouse)

56,626 United Nations, Economic and Social Commission for Western Asia, 1999: National farm data handbook for Lebanon. New York (GLIMS data warehouse)

768,403 Department of Veterinary Service, Malaysia: Selected Agricultural Indicators 2012 (Indikator Pertanian Terpilih 2012). Online data:

http://www.statistics.gov.my/portal/download_Agriculture/files/Selected_Agricultural_Indicators_Malays ia_2012.pdf

2,583,027 National Statistical Office of Mongolia (MONSIS): Number of livestock by type, by regions, soums, aimags and the Capital. Online data: http://www.1212.mn/en/contents/stats/contents_stat_fld_tree_html.jsp

13,981,114 Livestock Breeding and Veterinary Department (LBVD), Ministry of Livestock Breeding and Fisheries, Yangon, Myanmar: Myanmar Farm Animal Resources: Numbers and Distributions from Administrative Returns, 2011/12 (GLIMS data warehouse)

7,106,976 Ministry of Agriculture and Cooperatives (MOAC), Nepal: 2009 data (GLIMS data warehouse)

24,114,455 Agricultural Census Organization, Ministry of Food \& Agriculture, Pakistan: Agricultural Census 2010. Online data: http://www.pbs.gov.pk/content/agricultural-census-2010-pakistan-report

2,599,466 Bureau of Agricultural Statistics (BAS): Livestock Survey Philippines 2010 (GLIMS data warehouse)

9,671 Ministry of Municipal Affairs and Agriculture Department of Agriculture and Water Research: Agricultural Census 2000-2001 (GLIMS data warehouse) 


\begin{tabular}{|c|c|c|c|c|}
\hline Republic of Korea & 2011 & 1 & $3,353,353$ & Korea National Statistical Office: Korea Statistical Yearbook, 2012 (GLIMS data warehouse) \\
\hline $\begin{array}{l}\text { Republic of } \\
\text { Moldova }\end{array}$ & 2011 & 2 & 209,900 & $\begin{array}{l}\text { Statistica Moldovei: Statistical Yearbook of the Republic of Moldova 2011. Online data: } \\
\text { http://www.statistica.md/pageview.php?l=en\&idc }=263 \& i d=2193\end{array}$ \\
\hline Romania & 2011 & 1 & $1,988,939$ & $\begin{array}{l}\text { National Institute of Statistics, Romania: Tempo online database/Agriculture/Livestock and Animal } \\
\text { Production 2000-2011. Online data: https://statistici.insse.ro/shop/?lang=en }\end{array}$ \\
\hline Russian Federation & 2012 & 1 & $21,446,360$ & $\begin{array}{l}\text { Russian Federation - Federal State Statistical Service: Livestock and poultry production, } 2011 \text { and } 2012 . \\
\text { Online data: http://www.fedstat.ru/indicators/start.do }\end{array}$ \\
\hline Saudi Arabia & 2009 & 1 & 424,489 & $\begin{array}{l}\text { Central Department of Statistics and Information of Kingdom of Saudi Arabia: Statistical Yearbook, } 2010 \\
\text { Saudi Arabia (GLIMS data warehouse) }\end{array}$ \\
\hline Sri Lanka & 2012 & 2 & $1,235,535$ & $\begin{array}{l}\text { Agriculture and Environment Statistics Division, Department of Census and Statistics, Sri Lanka: Livestock } \\
\text { Population by Type and by District } 2003 \text { - 2012. Online data: } \\
\text { http://www.statistics.gov.lk/agriculture/Livestock/LivestockPopulationSubnational.html }\end{array}$ \\
\hline $\begin{array}{l}\text { Syrian Arab } \\
\text { Republic }\end{array}$ & 2004 & 1 & $1,024,120$ & $\begin{array}{l}\text { National Agricultural Policy Center - The Ministry of Agriculture and Agrarian Reform: Syrian agricultural } \\
\text { database (SAD) (GLIMS data warehouse) }\end{array}$ \\
\hline Tajikistan & 2009 & 2 & $1,834,727$ & Agency for Statistics, Tajikistan Republic: Regions of Tajikistan Republic, 2010 (GLIMS data warehouse) \\
\hline Thailand & 2006 & 1 & $6,003,883$ & $\begin{array}{l}\text { Regional Data Exchange System on Food and Agricultural Statistics in Asia and Pacific Countries: Thailand } \\
\text { sub national Statistics. Online data: http://www.faorap-apcas.org/thailand.html }\end{array}$ \\
\hline Turkey & 2009 & 2 & $10,723,958$ & Turkish Statistical Institute: Livestock statistics database 2009 (GLIMS data warehouse) \\
\hline Ukraine & 2012 & 1 & $4,645,900$ & State statistics committee Ukraine: Head of livestock and poultry by regions (GLIMS data warehouse) \\
\hline $\begin{array}{l}\text { United Arab } \\
\text { Emirates }\end{array}$ & 2000 & 1 & 96,050 & $\begin{array}{l}\text { Ministry of Agriculture and Fisheries: Agricultural Statistical Yearbook 2000. United Arab Emirates } \\
\text { (GLIMS data warehouse) }\end{array}$ \\
\hline Uzbekistan & 2002 & 1 & $5,416,100$ & $\begin{array}{l}\text { State Statistics Department of Uzbekistan: Agriculture of Uzbekistan, 2001. Minmakroeconomstat of } \\
\text { Republic Uzbekistan, State Statistics Department of Uzbekistan Tashkent, } 2002 \text { (GLIMS data warehouse) }\end{array}$ \\
\hline Viet Nam & 2001 & 6 & $3,710,385$ & $\begin{array}{l}\text { General Statistics Office of Vietnam: Agricultural Census database 2001. Online data: } \\
\text { http://www.gso.gov.vn/default_en.aspx?tabid=477\&idmid=4\&ItemID=1824 }\end{array}$ \\
\hline Yemen & 2007 & 1 & $1,394,707$ & $\begin{array}{l}\text { Directorate of Agricultural Statistics and Documentation, Ministry of Agriculture and Irrigation, Yemen } \\
\text { (GLIMS data warehouse) }\end{array}$ \\
\hline
\end{tabular}

No Data for: Bahrain, Brunei Darussalam, Christmas Island, Cocos (Keeling) Islands, Democratic People's Republic of Korea, Kuril Islands, Liancourt Rock, Maldives, Oman, Paracel Islands,

Scarborough Reef, Singapore, Senkaku Islands, Spratly Islands, Timor-Leste, Turkmenistan 


\section{Europe Tile}

\begin{tabular}{|c|c|c|c|c|}
\hline COUNTRY & YEAR & LEVEL & Totals in GLIMS & SOURCE \\
\hline Albania & 2010 & 1 & 493,000 & Institute of Statics, Albania: Agricultural sector, Livestock 2001-2010 (GLIMS data warehouse) \\
\hline Andorra & 2010 & 1 & 1,565 & $\begin{array}{l}\text { Ministry of Economy and Territory - Department of Agriculture, Andorra: Agriculture, Herding, Hunting } \\
\text { and Fishing - Livestock by type and parish 2003-2010 (GLIMS data warehouse) }\end{array}$ \\
\hline Austria & 2010 & 1 & $2,013,281$ & Statistics Austria: Livestock Survey, 2010 (GLIMS data warehouse) \\
\hline Belgium & 2010 & 3 & $1,878,963$ & $\begin{array}{l}\text { Directorate - General Statistics Belgium formerly known as NIS-INS: Data } 2010 \text { from William Wint, (all } \\
\text { acquired by Els Ducheyne at Avia_GIS, Zoersel, Belgium, process by William Wint, ERGO Ltd) (GLIMS } \\
\text { data warehouse) }\end{array}$ \\
\hline Croatia & 2003 & 1 & 398,037 & $\begin{array}{l}\text { Central Bureau of Statistics, Republic of Croatia: Agricultural Census 2003. Online data: } \\
\text { http://www.dzs.hr/default_e.htm }\end{array}$ \\
\hline Czech Republic & 2010 & 2 & $1,328,008$ & $\begin{array}{l}\text { Czech Statistical Office: Agrocensus regions: Farm Structure Survey and Survey on Agricultural Production } \\
\text { Methods 2010. Online data: http://www.czso.cz/csu/2012edicniplan.nsf/engp/2127-12 }\end{array}$ \\
\hline Denmark & 2010 & 2 & $1,571,050$ & $\begin{array}{l}\text { Denmark Statistics: Farms by region and selected farms, crops and livestock. Online data: } \\
\text { http://www.statistikbanken.dk/statbank5a/SelectVarVal/Define.asp?Maintable=BDF51\&PLanguage=1 }\end{array}$ \\
\hline Estonia & 2001 & 2 & 280,731 & $\begin{array}{l}\text { Statistikaamet Statistical Office of Estonia: Agricultural Census 2001. Online data: } \\
\text { http://www.stat.ee/agricultural-census-2001 }\end{array}$ \\
\hline Finland & 2010 & 6 & 925,800 & $\begin{array}{l}\text { The Information Centre of the Ministry of Agriculture and Forestry, Finland: Yearbook of Farm Statistics } \\
2011 \text { (GLIMS data warehouse) }\end{array}$ \\
\hline France & 2009 & 2 & $19,692,468$ & Service de la Statistique et de la Prospective (SSP): Statistique Agricole Annuelle (GLIMS data warehouse) \\
\hline Germany & 2007 & 2 & $12,351,818$ & $\begin{array}{l}\text { Federal Statistical Office, Germany: GENESIS database. Online data: } \\
\text { https://www.regionalstatistik.de/genesis/online/online;jsessionid=EE45147898822814978BE734145275C } \\
\text { 4?operation=sprachwechsel\&option=en }\end{array}$ \\
\hline Greece & 2007 & 2 & 731,996 & $\begin{array}{l}\text { Hellenic Statistic Authority (EL.STAT): Farms and number of animals by type, region and county (GLIMS } \\
\text { data warehouse) }\end{array}$ \\
\hline Hungary & 2010 & 1 & 684,000 & Hungarian Central Statistical Office: Regional Statistics. Online data: http://www.ksh.hu/agriculture \\
\hline Iceland & 2010 & 2 & 73,781 & Statistics Iceland: 2010 data (GLIMS data warehouse) \\
\hline Ireland & 2000 & 6 & $6,851,919$ & $\begin{array}{l}\text { Central Statistical Office Ireland: Census of Agriculture } 2000 \text { (data acquired by Els Ducheyne at Avia_GIS, } \\
\text { Zoersel, Belgium, process by William Wint, ERGO Ltd) (GLIMS data warehouse) (GLIMS data } \\
\text { warehouse) }\end{array}$ \\
\hline Italy & 2010 & 3 & $5,587,719$ & $\begin{array}{l}\text { Istituto Nazionale di Statistica: Censimento Agricoltura 2010. Online data: http://dati- } \\
\text { censimentoagricoltura.istat.it }\end{array}$ \\
\hline
\end{tabular}


Latvia

2001

Liechtenstein

Lithuania

Luxembourg

Malta

Montenegro

Netherlands

Norway

Poland

Portugal

Serbia

Slovakia

Slovenia

Spain

Sweden

Switzerland

The former

Yugoslav Republic

of Macedonia

United Kingdom
364,807 Central Statistical Bureau of Latvia: Results of 2001 Agricultural Census. Online data: http://www.csb.gov.lv/en/dati/agricultural-census-2001-30765.html

6,078 Amt für Statistik Liechtenstein: Landwirtschaftsstatistik 2009 (GLIMS data warehouse)

780,051 Lietuvos Statistikas Departamenta: Number of livestock by administrative territory, 2011 (GLIMS data warehouse)

196,295 Portail des statistiques du Grand-Duché de Luxembourg (STATEC): Recensement Agricole, 2008 (GLIMS data warehouse)

15,688 National Statistics Office of Malta: Census of Agriculture 2010. Online data: http://www.nso.gov.mt/statdoc/document_view.aspx?id=3287\&backurl=/themes/theme_page.aspx

78,633 Statistical office of Montenegro (MONSTAT): Agriculture Census 2010. Online data: http://www.monstat.org/eng/page.php?id=58\&pageid $=58$

3,975,194 Centraal Bureau voor de Statistiek, Netherlands: Agriculture: crops, livestock and land use by general far type, region - 2011 (GLIMS data warehouse)

867,641 Statistics Norway: Agriculture Statistics 2011 (GLIMS data warehouse)

5,700,000 Central Statistical Office, Poland: Statistical Yearbook of Agriculture 2010 (GLIMS data warehouse)

1,173,975 Instituto Nacional de Estatística - Statistics Portugal: Agricultural Census 2009. Online data: http://ra09.ine.pt/xportal/xmain?xpid=RA2009\&xpgid=ra_home

1,105,988 Statistical Office of Serbia: Municipalities of Serbia 2007 (GLIMS data warehouse)

471,965 Statistical Office of the Slovak Republic: Livestock by territory, products and period. Online data: http://px-web.statistics.sk/PXWebSlovak/DATABASE/En/databasetree.asp

471,584 Statistical Office of the Republic of Slovenia: Agricultural Census Slovenia 2010. Online data: http://www.stat.si/eng/novica_prikazi.aspx?id=4594

6,075,081 Ministerio de Medio Ambiente y Medio Rural y Marino, Gobierno de España (MARM): Encuestas Ganaderas 2010 (GLIMS data warehouse)

1,538,281 Statistics Sweden: Yearbook of Agricultural Statistics 2010 (GLIMS data warehouse)

1,597,484 Swiss Federal Statistical Office: Recensement des entreprises agricoles,1999-2009 (GLIMS data warehouse)

232,526 State Statistic Office of the Republic of Macedonia: Census of Agriculture 2007. Online data: http://www.stat.gov.mk/PrikaziPublikacija_en.aspx?id=51\&rbr=205

9,991,910 Office for National Statistics, U.K.: Statistics by Region. Online data: http://www.statistics.gov.uk/hub/regional-statistics/ 


\section{North America Tile}

\begin{tabular}{|c|c|c|c|c|}
\hline COUNTRY & YEAR & LEVEL & $\begin{array}{l}\text { Totals in } \\
\text { GLIMS }\end{array}$ & SOURCE \\
\hline Belize & 2003 & 1 & 57,800 & $\begin{array}{l}\text { Ministry Of Agriculture, Fisheries and Cooperatives, Belize: Annual Report 2003. Online data: } \\
\text { http://www.agriculture.gov.bz/PDF/Annual_Report_2003.pdf }\end{array}$ \\
\hline Canada & 2006 & 2 & $15,772,904$ & Statistics Canada: 2006 Census of Agriculture. Online data: http://www.statcan.gc.ca/ca-ra2006/index-eng.htm \\
\hline Cayman Islands & 2009 & 1 & 2,231 & $\begin{array}{l}\text { Economics and Statistics Office - Government of the Cayman Islands: Statistical Compendium } 2009 \text { (GLIMS } \\
\text { data warehouse) }\end{array}$ \\
\hline Costa Rica & 2000 & 1 & $1,358,209$ & Ministerio de Agricultura y Ganadería, Costa Rica: Censo Ganadero 2000 (GLIMS data warehouse) \\
\hline Cuba & 2009 & 1 & $3,586,303$ & $\begin{array}{l}\text { Oficina Nacional de Estadísticas, República de Cuba: Anuario Estadístico de Cuba } 2009 \text { - Edición } 2010 \text { (GLIMS } \\
\text { data warehouse) }\end{array}$ \\
\hline Dominican Republic & 1997 & 1 & $2,453,276$ & $\begin{array}{l}\text { Oficina Nacional de Estadística, Republica Dominicana: Republica Dominicana en cifras } 1997 \text { (GLIMS data } \\
\text { warehouse) }\end{array}$ \\
\hline El Salvador & 2007 & 2 & $1,141,323$ & Ministerio de Economía - El Salvador: IV Censo Agropecuario 2007-2008 (GLIMS data warehouse) \\
\hline Guadeloupe & 2000 & 1 & 64,236 & $\begin{array}{l}\text { Agreste la Statistique Agricole, Le Ministère de l'Agriculture, de l'Alimentation, de la Pêche et de la Ruralité, } \\
\text { France: Recensement Agricole 2000. Online data: http://agreste.agriculture.gouv.fr/recensement-agricole- } \\
\text { 2010/resultats-donnees-chiffrees/ }\end{array}$ \\
\hline Guatemala & 2003 & 2 & $1,627,522$ & $\begin{array}{l}\text { Instituto Nacional de Estadística, Guatemala: IV Censo Nacional Agropecuario, 2003. Online data: } \\
\text { http://www.ine.gob.gt/np/agropecuario/tomo\%20IV.pdf }\end{array}$ \\
\hline Honduras & 1993 & 1 & $2,077,460$ & $\begin{array}{l}\text { Secretaría de Planificación, Coordinación y Presupuesto, Honduras: IV Censo Nacional Agropecuario } 1993 \\
\text { (GLIMS data warehouse) }\end{array}$ \\
\hline Jamaica & 2009 & 1 & 113,338 & Agricultural Business Information System, Jamaica: Livestock Summary Report, 2009 (GLIMS data warehouse) \\
\hline Martinique & 2000 & 1 & 28,342 & $\begin{array}{l}\text { Agreste la Statistique Agricole, Le Ministère de l'Agriculture, de l'Alimentation, de la Pêche et de la Ruralité, } \\
\text { France: Recensement Agricole 2000. Online data: http://agreste.agriculture.gouv.fr/recensement-agricole- } \\
\text { 2010/resultats-donnees-chiffrees/ }\end{array}$ \\
\hline Mexico & 2007 & 2 & $23,237,877$ & $\begin{array}{l}\text { Instituto Nacional de Estadística Geografía e Informática, México: Censo Agrícola, Ganadero y Forestal } 2007 . \\
\text { Online data: } \\
\text { http://www.inegi.org.mx/est/contenidos/proyectos/Agro/ca2007/Resultados_Agricola/default.aspx }\end{array}$ \\
\hline Nicaragua & 2001 & 2 & $2,657,039$ & $\begin{array}{l}\text { Instituto Nacional de Estadística y Censos, Nicaragua: III Censo Nacional Agropecuario, } 2001 \text { (GLIMS data } \\
\text { warehouse) }\end{array}$ \\
\hline
\end{tabular}


Panama

Saint Lucia

Saint Vincent and

the Grenadines
1,531,914 Instituto Nacional de Estadística y Censo: Existencia de Animales en la República, por clase de animal, según provincia: 21 de abril de 1991 al 22 abril de 2001. Online data:

http://www.contraloria.gob.pa/inec/Avance/Avance.aspx?ID_CATEGORIA=2\&ID_CIFRAS=8\&ID_IDIO $\underline{\mathrm{MA}=1}$

259,918 National Agricultural Statistics Service - United States Department of Agriculture: National Agricultural Census 2007. Online data: http://www.agcensus.usda.gov/Publications/2007/Full_Report/

5,345 Ministry of Agriculture, Forestry and Fisheries: Census of Agriculture - Final report 2007. Online data: http://www.govt.lc/www/publications/AgricultureCensus2007.pdf

4,767 Ministry of Agriculture and Labour, Government of Saint Vincent and the Grenadines: National Agricultural Census 2000 (GLIMS data warehouse)

2007. Online data: http://www.agcensus usda.gov/Publications/2007/Full Report/

No Data for: Anguilla, Antigua and Barbuda, Bahamas, Barbados, Bermuda, Bird Island, British Virgin Islands, Dominica, Grenada, Haiti, Montserrat, Netherlands Antilles, Saint Kitts and Nevis,

Turks and Caicos Islands, United States Virgin Islands 


\section{South America Tile}

\begin{tabular}{|c|c|c|c|c|}
\hline COUNTRY & YEAR & LEVEL & $\begin{array}{l}\text { Totals in } \\
\text { GLIMS }\end{array}$ & SOURCE \\
\hline Argentina & 2009 & 2 & $53,637,537$ & $\begin{array}{l}\text { Instituto Nacional de Tecnología Agropecuaria, Argentina: Análisis de la actividad ganadera bovina de carne por } \\
\text { estratos de productores y composición del stock. Años } 2008 \text { y } 2009 \text { (GLIMS data warehouse) }\end{array}$ \\
\hline $\begin{array}{l}\text { Bolivia, } \\
\text { Plurinational State } \\
\text { of }\end{array}$ & 2008 & 1 & $8,189,599$ & $\begin{array}{l}\text { Instituto Nacional de Estadística, Bolivia: Encuesta Nacional Agropecuaria. Online data: } \\
\text { http://www.gobernacionlapaz.gob.bo/archivos/Sec_Deptal/SDPD/DID/Estadistica_Deptal/Agropecuario/R } \\
\text { esultados_ENA_2008/EncuestaNacional_Agropecuaria.pdf }\end{array}$ \\
\hline Brazil & 2009 & 2 & $205,225,804$ & $\begin{array}{l}\text { Istituto Brasileiro de Geografia e Estatistica: Producao da Pecuria Municipal 2009. Online data: } \\
\text { http://www.ibge.gov.br/home/estatistica/economia/ppm/2009/default.shtm }\end{array}$ \\
\hline Chile & 2007 & 3 & $3,718,532$ & $\begin{array}{l}\text { Instituto Nacional de Estadísticas, Chile: Censo Agropecuario y Forestal 2007. Online data: } \\
\text { http://www.ine.cl/canales/chile_estadistico/censos_agropecuarios/censo_agropecuario_07_comunas.php }\end{array}$ \\
\hline Colombia & 2009 & 1 & $22,787,372$ & Instituto Agropecuario, Colombia: Censo Agropecuario Nacional 2004 - 2009 (GLIMS data warehouse) \\
\hline Ecuador & 2000 & 2 & $4,481,052$ & $\begin{array}{l}\text { Project SICA, Agricultural Information System, Ministry of Agriculture and Livestock, Ecuador: III Censo } \\
\text { Nacional Agropecuario (GLIMS data warehouse) }\end{array}$ \\
\hline $\begin{array}{l}\text { Falkland Islands } \\
\text { (Malvinas) }\end{array}$ & 2010 & 1 & 4,738 & Department of Agriculture, Falkland Islands Government: Statistics Book 2010 (GLIMS data warehouse) \\
\hline French Guiana & 2000 & 6 & 9,394 & $\begin{array}{l}\text { Agreste la Statistique Agricole, Le Ministère de l'Agriculture, de l'Alimentation, de la Pêche et de la Ruralité, } \\
\text { France: Recensement Agricole 2000. Online data: http://agreste.agriculture.gouv.fr/recensement-agricole- } \\
\text { 2010/resultats-donnees-chiffrees/ }\end{array}$ \\
\hline Paraguay & 2008 & 2 & $10,466,641$ & Ministerio de Agricultura y Ganadería, Paraguay: Censo Agropecuario Nacional 2008 (GLIMS data warehouse) \\
\hline Peru & 2010 & 1 & $5,520,197$ & Instituto Nacional de Estadística e Informática, Peru: Compendio Estadístico 2011 (GLIMS data warehouse) \\
\hline Suriname & 1994 & 1 & 123,630 & $\begin{array}{l}\text { Environmental Research Group Oxford, ERGO: ERGO, } 1996 \text { "Livestock Geography. A demonstration of GIS } \\
\text { techniques applied to Global Livestock Systems and Populations", Animal Health Division, FAO, Rome } \\
\text { (William Wint extracted from the agroecological zone estimates derived from the FAOSTAT data for 1994) } \\
\text { (GLIMS data warehouse) }\end{array}$ \\
\hline Uruguay & 2010 & 1 & $11,092,285$ & $\begin{array}{l}\text { Ministerio de Ganadería, Agricultura y Pesca, Estadísticas Agropecuarias, Uruguay: Datos de la Declaración } \\
\text { Jurada de DICOSE } 2010 \text { - Datos Generales de Lechería y de Suinos (GLIMS data warehouse) }\end{array}$ \\
\hline $\begin{array}{l}\text { Venezuela, } \\
\text { Bolivarian Republic }\end{array}$ & 2007 & 2 & $13,302,976$ & $\begin{array}{l}\text { Ministerio del Poder Popular para la Agricultura y Tierras - Venezuela: VII Censo Agrícola 2007. Online data: } \\
\text { http://censo.mat.gob.ve/ }\end{array}$ \\
\hline
\end{tabular}




\section{Oceania Tile}

\begin{tabular}{|c|c|c|c|c|}
\hline COUNTRY & YEAR & LEVEL & $\begin{array}{l}\text { Totals in } \\
\text { GLIMS }\end{array}$ & SOURCE \\
\hline American Samoa & 2003 & 1 & 300 & $\begin{array}{l}\text { National Agricultural Statistics Service - United States Department of Agriculture: National Agricultural Census } \\
\text { 2007. Online data: http://www.agcensus.usda.gov/Publications/2007/Full_Report/ }\end{array}$ \\
\hline Australia & 2007 & 6 & $26,113,333$ & $\begin{array}{l}\text { Australian Bureau of Statistics: Agricultural commodities: small area data, Australia 2006-2007 (GLIMS data } \\
\text { warehouse) }\end{array}$ \\
\hline New Zealand & 2010 & 1 & $9,617,244$ & $\begin{array}{l}\text { Statistics New Zealand: } 2011 \text { Livestock numbers by Regional Council. Online data: } \\
\text { http://www.stats.govt.nz/tools_and_services/nzdotstat/agriculture-statistics.aspx }\end{array}$ \\
\hline $\begin{array}{l}\text { Northern Mariana } \\
\text { Islands }\end{array}$ & 2007 & 1 & 1,395 & $\begin{array}{l}\text { National Agricultural Statistics Service - United States Department of Agriculture: National Agricultural Census } \\
\text { 2007. Online data: http://www.agcensus.usda.gov/Publications/2007/Full_Report/ }\end{array}$ \\
\hline Papua New Guinea & 1989 & 1 & 62,888 & National Statistical Office: Agricultural Large Holdings 1989 (GLIMS data warehouse) \\
\hline Tonga & 2001 & 1 & 10,354 & $\begin{array}{l}\text { Ministry of Agriculture and Forestry, Kingdom of Tonga: Agriculture Census 2001. Online data: } \\
\text { http://www.spc.int/prism/tonga/index.php/surveys/agriculture-census-2011 }\end{array}$ \\
\hline
\end{tabular}

Pitcairn, Samoa, Solomon Islands, Tokelau, Tuvalu, Vanuatu, Wake Island, Wallis and Futun 
Supplementary information $5 \mathrm{~b}$ - Country details sub-national statistics for pigs (year and level of observation) by continental tile.

\section{Africa Tile}

\begin{tabular}{|c|c|c|c|c|}
\hline COUNTRY & YEAR & LEVEL & $\begin{array}{l}\text { Totals in } \\
\text { GLIMS }\end{array}$ & SOURCE \\
\hline Algeria & 2002 & 1 & 5,702 & $\begin{array}{l}\text { Direction des Services Vétérinaires - Ministère de l'Agriculture et du Développement Rural } 2003 \text { (GLIMS data } \\
\text { warehouse) }\end{array}$ \\
\hline Angola & 2009 & 1 & $3,984,614$ & $\begin{array}{l}\text { CountrySTAT - Food and agriculture data network: Número de animais vivos by Ano, Especie, Nivel } \\
\text { administrativo. Online data: http:// countrystat.org/home.aspx?c=AGO\&ta=007SPD035\&tr=21 }\end{array}$ \\
\hline Benin & 2008 & 2 & 329,700 & $\begin{array}{l}\text { CountrySTAT - Food and agriculture data network: Élevage - Total des effectifs d'animaux vivants 2010-2012. } \\
\text { Online data: http://www.countrystat.org/home.aspx?c=BEN\&ta=053SPD135\&tr=21 }\end{array}$ \\
\hline Botswana & 2009 & 2 & 3,302 & Central Statistics Office of Botswana: 2009 and 2010 Annual Agricultural Surveys (GLIMS data warehouse) \\
\hline Burkina Faso & 2008 & 2 & $2,083,127$ & $\begin{array}{l}\text { Ministère de l'Economie et de Développement, Direction General des Prévisions et des Statistiques Agricoles: } \\
\text { Les régions en chiffres, } 2008 \text { \& } 2010 \text { (GLIMS data warehouse) }\end{array}$ \\
\hline Burundi & 2005 & 2 & 156,138 & $\begin{array}{l}\text { Direction Provinciale de l'Agriculture et de l'Élevage, Burundi: Monographies des les Communes } 2006 \text { (GLIMS } \\
\text { data warehouse)) }\end{array}$ \\
\hline Cameroon & 2007 & 2 & 218,080 & CountrySTAT - Food and agriculture data network (GLIMS data warehouse) \\
\hline Cape Verde & 2004 & 2 & 77,316 & Directorate of livestock, Cape Verde: Recenseamento Geral da Agricultura, 2004 (GLIMS data warehouse) \\
\hline $\begin{array}{l}\text { Central African } \\
\text { Republic }\end{array}$ & 1990 & 1 & 441,480 & $\begin{array}{l}\text { Environmental Research Group Oxford, ERGO: FAO, 2004. Agro-ecological distributions for Africa (GLIMS } \\
\text { data warehouse) }\end{array}$ \\
\hline Chad & 2007 & 1 & 39,220 & $\begin{array}{l}\text { Direction de l'Élevage et des Ressources Animales: Effectifs du cheptel national par région administrative } 2007 \\
\text { (GLIMS data warehouse) }\end{array}$ \\
\hline Congo & 2000 & 1 & 15,455 & $\begin{array}{l}\text { Ministère de l'Agriculture et de l'Élevage: Enquête Agricole Pilote, Campagne Agricole 1999-2000 (GLIMS data } \\
\text { warehouse) }\end{array}$ \\
\hline Côte d'Ivoire & 2001 & 1 & 476,708 & $\begin{array}{l}\text { Ministère de l'Agriculture et des Ressources Animales, Direction de la Programmation, Côte d'Ivoire: } \\
\text { Recensement National de l'Agriculture } 2001 \text { (GLIMS data warehouse) }\end{array}$ \\
\hline $\begin{array}{l}\text { Democratic } \\
\text { Republic of the } \\
\text { Congo }\end{array}$ & 2009 & 1 & 960,305 & $\begin{array}{l}\text { Centre for Ticks and Tick-Borne Diseases: Preliminary census data from SADC and Eastern Africa (GLIMS data } \\
\text { warehouse) }\end{array}$ \\
\hline Eritrea & 2002 & 2 & 2,203 & $\begin{array}{l}\text { Environmental Research Group Oxford, ERGO: GIEWS special reports. Online data: } \\
\text { http://www.fao.org/docrep/005/y } 7678 \mathrm{e} / \mathrm{y} 7678 \mathrm{e} 00 . \mathrm{htm}\end{array}$ \\
\hline Ethiopia & 2002 & 3 & 28,598 & Central Statistical Authority of Ethiopia: Agricultural Census 2001-2002 (GLIMS data warehouse) \\
\hline Gabon & 2002 & 1 & 213,000 & $\begin{array}{l}\text { Environmental Research Group Oxford, ERGO: FAO, 2004. Agro-ecological distributions for Africa (GLIMS } \\
\text { data warehouse) }\end{array}$ \\
\hline
\end{tabular}




\begin{tabular}{|c|c|}
\hline Gambia & 2007 \\
\hline Ghana & 1996 \\
\hline Guinea & 2002 \\
\hline Guinea-Bissau & 1994 \\
\hline Kenya & 2005 \\
\hline Lesotho & 2008 \\
\hline Madagascar & 2002 \\
\hline Malawi & 2007 \\
\hline Mali & 2010 \\
\hline Mauritius & 2010 \\
\hline Morocco & 2002 \\
\hline Mozambique & 2010 \\
\hline Namibia & 2006 \\
\hline Niger & 2002 \\
\hline Nigeria & 2007 \\
\hline Réunion & 2000 \\
\hline Rwanda & 2008 \\
\hline Saint Helena & 2009 \\
\hline Sao Tome and & 1997 \\
\hline Principe & \\
\hline Senegal & 2009 \\
\hline
\end{tabular}

24,281 National Agriculture Development Agency, Gambia: National Agricultural Sample Survey Report (NASS), Oct. 2008 (GLIMS data warehouse)

354,678 Veterinary Services of Ghana: Livestock Census, 1994-1996 (GLIMS data warehouse)

97,205 Service National des Statistiques Agricoles, République de Guinée 2002 (GLIMS data warehouse)

204,375 Instituto Nacional de Estadistica: Inquerito Annual sobre Superficie Rendimento e Producao. Campanha 93/94 (GLIMS data warehouse)

310,154 Ministry of Livestock Development, Kenya: Compilation of divisional data 2005 (GLIMS data warehouse)

83,976 Bureau of Statistics of Lesotho: Livestock winter 2008/09 Tables. Online data: http://www.bos.gov.ls/Downloads.htm

460,805 Direction des Ressources Animales: Situation de l'élevage a Madagascar 2002 (GLIMS data warehouse)

792,364 Agriculture Statistics Division of the National Statistical Office of Malawi (NSO): National Census of Agriculture and Livestock (October 2006 - October 2007) (GLIMS data warehouse)

75,089 CountrySTAT - Food and agriculture data network: Élevage - Total des effectifs d'animaux vivants. Online data: http://www.countrystat.org/home.aspx?c=MLI\&tr=21

22,327 Central Statistic Office of Mauritius: Digest of Agricultural Statistics (July 2010) (GLIMS data warehouse)

4,783 Ministère de l'Agriculture, du Développement Rural et des Pêches Maritimes: Evolution des effective au niveau des provinces (GLIMS data warehouse)

1,340,712 National Directorate of Livestock: Censo Agropecuario 2009-2010 (GLIMS data warehouse)

52,485 FAO Special Report, FAO/WFP Crop, Livestock and Food Security Assessment Mission to Namibia (GLIMS data warehouse)

39,000 Ministère des Ressources Animales: Revue des statistiques courantes sur l'élevage au Niger (GLIMS data warehouse)

2,444,096 National Bureau of Statistics, Nigeria: Agricultural Census 2007 (GLIMS data warehouse)

70,921 Agreste la Statistique Agricole, Le Ministère de l'Agriculture, de l'Alimentation, de la Pêche et de la Ruralité France: Recensement Agricole 2000. Online data: http://agriculture.gouv.fr/recensement-agricole-2010

310,834 National Institute of Statistics of Rwanda: National Agricultural Survey 2008 (Final report) (GLIMS data warehouse)

773 Agriculture and Natural Resources Department of St. Helena: Livestock Census 2009 (GLIMS data warehouse)

13,495 Ministère de l'Agriculture et de la Pèche: Enquête Agricole de Production 1997 (GLIMS data warehouse)

344,172 Agence Nationale de la Statistique et de la Démographie du Sénégal: Live Data Base (LDB). Online data: http://www.ansd.sn/publications_SES_region.html

65,423 Ministry of Agriculture \& Food Security of Sierra Leone: 2004 Populations and Housing Census Statistical Office (GLIMS data warehouse) 
Somalia

South Africa

Swaziland

Togo

Uganda

United Republic of

Tanzania

Zambia

Zimbabwe
2002

2002

2010

2002

2000

2008

2009

2008
4,002 Environmental Research Group Oxford, ERGO: FAO, 2004. Agro-ecological distributions for Africa (GLIMS data warehouse)

1,979,931 Directorate Veterinary Services, South Africa: Animal Disease Report, January to September 2003 (GLIMS data warehouse)

38,915 Directorate of Veterinary and Livestock Services: Swaziland Livestock Statistics, Census 2010 (GLIMS data warehouse)

243,241 Data from Dr. Guy Hendrickx (GLIMS data warehouse)

770,319 Uganda Bureau of Statistics: Uganda Census 2000 (GLIMS data warehouse)

1,592,600 CountrySTAT - Food and agriculture data network (GLIMS data warehouse)

517,553 Department of Research and Specialist Services, Zambia: National Livestock Census by Province 1994 to 2009 (GLIMS data warehouse)

No Data for: Bassas da India, British Indian Ocean Territory, Comoros, Djibouti, Egypt, Equatorial Guinea, Europa Island, Glorioso Island, Juan de Nova Island, Liberia, Libya, Mauritania, Mayotte, Seychelles, Sudan, Tromelin Island, Tunisia, Western Sahara 


\section{Asia Tile}

\begin{tabular}{|c|c|c|c|c|}
\hline COUNTRY & YEAR & LEVEL & Totals in GLIMS & SOURCE \\
\hline Armenia & 2011 & 1 & 114,777 & $\begin{array}{l}\text { National statistical service of the republic of Armenia: Atlas the Republic of Armenia by Regions and the } \\
\text { Yerevan City, 2011. Online data: http://www.armstat.am/en/?nid=50 }\end{array}$ \\
\hline Azerbaijan & 2011 & 1 & 6,146 & $\begin{array}{l}\text { The State Statistical Committee of the Republic of Azerbaijan: The data on regions. Online data: } \\
\text { http://www.stat.gov.az/source/agriculture/indexen.php }\end{array}$ \\
\hline Bangladesh & 2009 & 2 & 206,872 & FAO - Emergency Center for Transboundary Animal Diseases (GLIMS data warehouse) \\
\hline Belarus & 2011 & 2 & $2,954,600$ & $\begin{array}{l}\text { National Statistical Committee of the Republic of Belarus: Agriculture of the Republic of Belarus, } 2011 \\
\text { (GLIMS data warehouse) }\end{array}$ \\
\hline Bhutan & 2009 & 2 & 21,532 & $\begin{array}{l}\text { Ministry of agriculture and forest, Royal government of Bhutan: Dzongkhag livestock sector (GLIMS data } \\
\text { warehouse) }\end{array}$ \\
\hline Bulgaria & 2010 & 6 & 664,000 & $\begin{array}{l}\text { Ministry of Agriculture and Forestry - Agrostatistics Directorate, Bulgaria: Agricultural Census 2010. Online } \\
\text { data: } \\
\text { http://www.mzh.government.bg/MZH/ShortLinks/SelskaPolitika/Agrostatistics/Structure_agricultural_h } \\
\text { oldings/Results.aspx }\end{array}$ \\
\hline Cambodia & 2011 & 2 & $2,076,990$ & Ministry of Agriculture, Forestry and Fisheries: Cambodia Livestock Census 2011 (GLIMS data warehouse) \\
\hline China, mainland & 2006 & 3 & $510,417,525$ & $\begin{array}{l}\text { National Bureau of Statistics of China: China Statistical Yearbook, 2006. For Taiwan: Statistics Office, } \\
\text { COA, Executive Yuan, Taiwan Statistical Yearbook, } 2010 \text { (GLIMS data warehouse) }\end{array}$ \\
\hline Cyprus & 2003 & 1 & 443,040 & $\begin{array}{l}\text { Statistical Service of Cyprus: Census of Agriculture 2003. Online data: } \\
\text { http://www.mof.gov.cy/mof/cystat/statistics.nsf/All/DE20AEA98C4DD6EEC22577A50041F0B8/\$file } \\
\text { /Census_of_Agriculture-2003.pdf?OpenElement }\end{array}$ \\
\hline Georgia & 2005 & 2 & 480,695 & $\begin{array}{l}\text { AgroWeb Central Asia and Caucasus: Georgian National Association for Animal Production (GNAAP), } \\
2005 \text { (GLIMS data warehouse) }\end{array}$ \\
\hline India & 2007 & 2 & $10,501,506$ & $\begin{array}{l}\text { Department of Animal Husbandry, Dairying \& Fisheries, Ministry of Agriculture, India: Census of } \\
\text { Agriculture, } 2007 \text { (GLIMS data warehouse) }\end{array}$ \\
\hline Indonesia & 2011 & 1 & $5,854,781$ & Department Pertanian Republik Indonesia: Buku Statistik, 2012 (GLIMS data warehouse) \\
\hline Japan & 2009 & 1 & $9,902,400$ & $\begin{array}{l}\text { Statistics Department, Minister's Secretariat, Ministry of Agriculture, Forestry and Fisheries: Japan } \\
\text { Statistical Yearbook 2010. Online data: } \\
\underline{\text { http://www.maff.go.jp/e/tokei/kikaku/nenji_e/85nenji/index.html }}\end{array}$ \\
\hline Kazakhstan & 2004 & 1 & $1,341,300$ & $\begin{array}{l}\text { Agency on Statistics of the Republic of Kazakhstan: Main socio economic indicators of regions in } \\
\text { Kazakhstan 2005. Almaty, Kazakhstan (GLIMS data warehouse) }\end{array}$ \\
\hline Kyrgyzstan & 2003 & 1 & 105,476 & $\begin{array}{l}\text { National Statistic Committee of Kyrgyzstan: Results of the first agricultural census of the Kyrgyz Republic } \\
\text { of } 2003 \text { (Second Stage) (GLIMS data warehouse) }\end{array}$ \\
\hline
\end{tabular}




\begin{tabular}{|c|c|}
\hline Lao People's & 2009 \\
\hline Democratic & \\
\hline Republic & \\
\hline Malaysia & 2001 \\
\hline Myanmar & 2011 \\
\hline Nepal & 2009 \\
\hline Philippines & 2010 \\
\hline Republic of Korea & 2005 \\
\hline $\begin{array}{l}\text { Republic of } \\
\text { Moldova }\end{array}$ & 2010 \\
\hline Romania & 2011 \\
\hline Russian Federation & 2011 \\
\hline Sri Lanka & 2011 \\
\hline Tajikistan & 2009 \\
\hline Thailand & 2006 \\
\hline Turkey & 2009 \\
\hline Ukraine & 2011 \\
\hline Uzbekistan & 2001 \\
\hline Viet Nam & 2001 \\
\hline
\end{tabular}

2,663,363 Department of Livestock and Fisheries, Laos: 2009 district data (David Bourn, “ Environmental Animal Health Management Initiative for Enhanced Smallholder Production”, 2010) (GLIMS data warehouse)

2,038,278 Department of Veterinary Service, Malaysia: Livestock Products statistics 1996-2002 (GLIMS data warehouse)

10,248,983 Livestock Breeding and Veterinary Department (LBVD), Ministry of Livestock Breeding and Fisheries, Yangon, Myanmar: Myanmar Farm Animal Resources: Numbers and Distributions from Administrative Returns, 2011/12 (GLIMS data warehouse)

1,021,122 Ministry of Agriculture and Cooperatives (MOAC), Nepal: 2009 data (GLIMS data warehouse)

12,793,664 Bureau of Agricultural Statistics (BAS): Livestock Survey Philippines 2010 (GLIMS data warehouse)

8,961,505 Ministry of Agriculture \& Forestry. Republic of Korea: Agricultural and Forestry Statistical Yearbook 2005 (GLIMS data warehouse)

377,100 Statistica Moldovei: Livestock in all categories of producers in territorial aspect, 2010. Online data: http:/ / www.statistica.md/pageview.php?l=en\&idc=349

5,363,797 National Institute of Statistics, Romania: Tempo online database/Agriculture/Livestock and Animal Production 2000-2011. Online data: https://statistici.insse.ro/shop/?lang=en

18,634,550 Russian Federation - Federal State Statistical Service: Livestock and poultry production, 2011 and 2012. Online data: http://www.fedstat.ru/indicators/start.do

82,030 Agriculture and Environment Statistics Division, Department of Census and Statistics, Sri Lanka: Livestock Population by Type and by District - 2003 - 2012. Online data: http://www.statistics.gov.lk/agriculture/Livestock/LivestockPopulationSubnational.html

419 Agency for Statistics, Tajikistan Republic: Regions of Tajikistan Republic, 2010 (GLIMS data warehouse)

11,763,705 Regional Data Exchange System on Food and Agricultural Statistics in Asia and Pacific Countries: Thailand sub national Statistics. Online data: http://www.faorap-apcas.org/thailand.html

1,896 Turkish Statistical Institute: Livestock statistics database 2009 (GLIMS data warehouse)

7,960,400 State statistics committee Ukraine: Head of livestock and poultry by regions (GLIMS data warehouse)

81,600 State Statistics Department of Uzbekistan: Agriculture of Uzbekistan, 2001. Minmakroeconomstat of Republic Uzbekistan, State Statistics Department of Uzbekistan Tashkent, 2002 (GLIMS data warehouse)

21,445,693 General Statistics Office of Vietnam: Agricultural Census database 2001. Online data: http://www.gso.gov.vn/default_en.aspx?tabid=477\&idmid=4\&ItemID=1824

No Data for: Afghanistan, Bahrain, Brunei Darussalam, Christmas Island, Cocos (Keeling) Islands, Democratic People's Republic of Korea, Islamic Republic of Iran, Iraq, Israel, Jordan, Kuril Islands, Kuwait, Lebanon, Liancourt Rock, Maldives, Mongolia, Oman, Pakistan, Paracel Islands, Qatar, Saudi Arabia, Scarborough Reef, Senkaku Islands, Singapore, Spratly Islands, Syrian Arab Republic,

Timor-Leste, Turkmenistan, United Arab Emirates, Yemen 


\section{Europe Tile}

\begin{tabular}{|c|c|c|c|c|}
\hline COUNTRY & YEAR & LEVEL & Totals in GLIMS & SOURCE \\
\hline Albania & 2010 & 1 & 164,500 & Institute of Statics, Albania: Agricultural sector, Livestock 2001-2010 (GLIMS data warehouse) \\
\hline Austria & 2010 & 1 & $3,134,156$ & Statistics Austria: Livestock Survey, 2010 (GLIMS data warehouse) \\
\hline Belgium & 2010 & 2 & $6,429,600$ & $\begin{array}{l}\text { EUROSTAT online database. General Statistics, Region, Agriculture, Animal Populations and Land Use. } \\
\text { Online data: http://epp.eurostat.ec.europa.eu/portal/page/portal/agriculture/data/ }\end{array}$ \\
\hline Bosnia and & 2009 & 6 & 529,095 & Federal Office of Statistics of Federation of Bosnia and Herzegovina: Number of animals from Federal \\
\hline Herzegovina & & & & Agro-Mediterranean Institute of Mostar (GLIMS data warehouse) \\
\hline Croatia & 2003 & 1 & $1,726,895$ & $\begin{array}{l}\text { Central bureau of statistics, Republic of Croatia: Agricultural Census 2003. Online data: } \\
\text { http://www.dzs.hr/default_e.htm }\end{array}$ \\
\hline Czech Republic & 2010 & 2 & $1,906,929$ & $\begin{array}{l}\text { Czech Statistical Office: Agrocensus regions: Farm Structure Survey and Survey on Agricultural Production } \\
\text { Methods 2010. Online data: http://www.czso.cz/csu/2012edicniplan.nsf/engp/2127-12 }\end{array}$ \\
\hline Denmark & 2010 & 2 & $13,173,060$ & $\begin{array}{l}\text { Denmark Statistics: Farms by region and selected farms, crops and livestock. Online data: } \\
\text { http://www.statistikbanken.dk/statbank5a/SelectVarVal/Define.asp?Maintable=BDF51\&PLanguage=1 }\end{array}$ \\
\hline Estonia & 2001 & 2 & 327,195 & $\begin{array}{l}\text { Statistikaamet Statistical Office of Estonia: Agricultural Census 2001. Online data: } \\
\text { http://www.stat.ee/agricultural-census-2001 }\end{array}$ \\
\hline Finland & 2010 & 6 & $1,366,800$ & $\begin{array}{l}\text { The Information Centre of the Ministry of Agriculture and Forestry, Finland: Yearbook of Farm Statistics } \\
2011 \text { (GLIMS data warehouse) }\end{array}$ \\
\hline France & 2009 & 2 & $13,245,288$ & Service de la Statistique et de la Prospective (SSP): Statistique Agricole Annuelle (GLIMS data warehouse) \\
\hline Germany & 2007 & 2 & $25,247,881$ & $\begin{array}{l}\text { Federal Statistical Office, Germany: GENESIS database. Online data: } \\
\text { https://www.regionalstatistik.de/genesis/online/online;jsessionid=EE45147898822814978BE734145275C } \\
\text { 4?operation=sprachwechsel\&option=en }\end{array}$ \\
\hline Greece & 2007 & 2 & $1,111,540$ & $\begin{array}{l}\text { Hellenic Statistic Authority (EL.STAT): Farms and number of animals by type, region and county (GLIMS } \\
\text { data warehouse) }\end{array}$ \\
\hline Hungary & 2010 & 1 & $3,169,000$ & Hungarian Central Statistical Office: Regional Statistics. Online data: http://www.ksh.hu/agriculture \\
\hline Iceland & 2010 & 2 & 3,615 & Statistics Iceland: 2010 data (GLIMS data warehouse) \\
\hline Ireland & 2000 & 1 & $1,722,108$ & $\begin{array}{l}\text { Central Statistical Office Ireland: Census of Agriculture 2000. Online data: } \\
\underline{\text { http://www.cso.ie/en/releasesandpublications/agricultureandfishing/censusofagriculturemainresultsjune } 20} \\
\underline{00 /}\end{array}$ \\
\hline Italy & 2010 & 3 & $9,331,143$ & $\begin{array}{l}\text { Istituto Nazionale di Statistica: Censimento Agricoltura 2010. Online data: http://dati- } \\
\text { censimentoagricoltura.istat.it }\end{array}$ \\
\hline Latvia & 2001 & 1 & 368,909 & $\begin{array}{l}\text { Central Statistical Bureau of Latvia: Results of } 2001 \text { Agricultural Census. Online data: } \\
\text { http://www.csb.gov.lv/en/dati/agricultural-census-2001-30765.html }\end{array}$ \\
\hline
\end{tabular}




\begin{tabular}{|c|c|c|c|c|}
\hline Liechtenstein & 2009 & 6 & 1,811 & Amt für Statistik Liechtenstein: Landwirtschaftsstatistik 2009 (GLIMS data warehouse) \\
\hline Lithuania & 2010 & 2 & 942,163 & $\begin{array}{l}\text { Lietuvos Statistikas Departamenta: Number of livestock by administrative territory, } 2011 \text { (GLIMS data } \\
\text { warehouse) }\end{array}$ \\
\hline Luxembourg & 2008 & 2 & 77,841 & $\begin{array}{l}\text { Portail des statistiques du Grand-Duché de Luxembourg (STATEC): Recensement Agricole, } 2008 \text { (GLIMS } \\
\text { data warehouse) }\end{array}$ \\
\hline Malta & 2010 & 6 & 70,593 & $\begin{array}{l}\text { National Statistics Office of Malta: Census of Agriculture 2010. Online data: } \\
\text { http://www.nso.gov.mt/statdoc/document_view.aspx?id=3287\&backurl=/themes/theme_page.aspx }\end{array}$ \\
\hline Montenegro & 2010 & 1 & 41,118 & $\begin{array}{l}\text { Statistical office of Montenegro (MONSTAT): Agriculture Census 2010. Online data: } \\
\text { http://www.monstat.org/eng/page.php?id=58\&pageid=58 }\end{array}$ \\
\hline Netherlands & 2010 & 2 & $12,254,972$ & $\begin{array}{l}\text { Centraal Bureau voor de Statistiek, Netherlands: Agriculture: crops, livestock and land use by general far } \\
\text { type, region - } 2011 \text { (GLIMS data warehouse) }\end{array}$ \\
\hline Norway & 2010 & 1 & $1,607,772$ & Statistics Norway: Agriculture Statistics 2011 (GLIMS data warehouse) \\
\hline Poland & 2009 & 1 & $14,278,600$ & Central Statistical Office, Poland: Statistical Yearbook of Agriculture 2010 (GLIMS data warehouse) \\
\hline Portugal & 2009 & 2 & $1,851,290$ & $\begin{array}{l}\text { Instituto Nacional de Estatística - Statistics Portugal: Agricultural Census 2009. Online data: } \\
\text { http://ra09.ine.pt/xportal/xmain?xpid=RA2009\&xpgid=ra_home }\end{array}$ \\
\hline Serbia & 2006 & 1 & $3,998,927$ & Statistical Office of Serbia: Municipalities of Serbia 2007 (GLIMS data warehouse) \\
\hline Slovakia & 2009 & 2 & 740,862 & $\begin{array}{l}\text { Statistical Office of the Slovak Republic: Livestock by territory, products and period. Online data: } \\
\text { http://px-web.statistics.sk/PXWebSlovak/DATABASE/En/databasetree.asp }\end{array}$ \\
\hline Slovenia & 2010 & 2 & 308,540 & $\begin{array}{l}\text { Statistical Office of the Republic of Slovenia: Agricultural Census Slovenia 2010. Online data: } \\
\text { http://www.stat.si/eng/novica_prikazi.aspx?id=4594 }\end{array}$ \\
\hline Spain & 2010 & 2 & $25,704,041$ & $\begin{array}{l}\text { Ministerio de Medio Ambiente y Medio Rural y Marino, Gobierno de España (MARM): Encuestas } \\
\text { Ganaderas, } 2010 \text { (GLIMS data warehouse) }\end{array}$ \\
\hline Sweden & 2009 & 1 & $1,528,740$ & Statistics Sweden: Yearbook of Agricultural Statistics 2010 (GLIMS data warehouse) \\
\hline Switzerland & 2009 & 1 & $1,557,204$ & Swiss Federal Statistical Office: Recensement des entreprises agricoles,1999-2009 (GLIMS data warehouse) \\
\hline $\begin{array}{l}\text { The former } \\
\text { Yugoslav Republic } \\
\text { of Macedonia }\end{array}$ & 2006 & 2 & 173,185 & $\begin{array}{l}\text { State Statistic Office of the Republic of Macedonia: Census of Agriculture 2007. Online data: } \\
\text { http://www.stat.gov.mk/PrikaziPublikacija_en.aspx?id=51\&rbr=205 }\end{array}$ \\
\hline United Kingdom & 2009 & 2 & $4,525,710$ & $\begin{array}{l}\text { Office for National Statistics, U.K.: Statistics by Region. Online data: } \\
\text { http://www.statistics.gov.uk/hub/regional-statistics/ }\end{array}$ \\
\hline
\end{tabular}

No Data for: Andorra, Faroe Islands, Gibraltar, Guernsey, Holy See, Isle of Man, Jersey, Madeira Islands, Monaco, San Marino, Svalbard and Jan Mayen Islands 


\section{North America Tile}

\begin{tabular}{|c|c|c|c|c|}
\hline COUNTRY & YEAR & LEVEL & $\begin{array}{l}\text { Totals in } \\
\text { GLIMS }\end{array}$ & SOURCE \\
\hline Belize & 2003 & 1 & 21,224 & $\begin{array}{l}\text { Ministry Of Agriculture, Fisheries and Cooperatives, Belize: Annual Report 2003. Online data: } \\
\text { http://www.agriculture.gov.bz/PDF/Annual_Report_2003.pdf }\end{array}$ \\
\hline Canada & 2006 & 2 & $14,168,313$ & Statistics Canada: 2006 Census of Agriculture. Online data: http://www.statcan.gc.ca/ca-ra2006/index-eng.htm \\
\hline Cayman Islands & 2009 & 1 & 1,063 & $\begin{array}{l}\text { Economics and Statistics Office - Government of the Cayman Islands: Statistical Compendium } 2009 \text { (GLIMS } \\
\text { data warehouse) }\end{array}$ \\
\hline Costa Rica & 2006 & 1 & 297,359 & $\begin{array}{l}\text { Ministerio de Agricultura y Ganadería, Costa Rica: Programa Nacional de Sanidad Porcina } 2006 \text { (GLIMS data } \\
\text { warehouse) }\end{array}$ \\
\hline El Salvador & 2007 & 2 & 165,866 & Ministerio de Economía - El Salvador: IV Censo Agropecuario 2007-2008 (GLIMS data warehouse) \\
\hline Guadeloupe & 2000 & 1 & 25,839 & $\begin{array}{l}\text { Agreste la Statistique Agricole, Le Ministère de l'Agriculture, de l'Alimentation, de la Pêche et de la Ruralité, } \\
\text { France: Recensement Agricole 2000. Online data: http://agreste.agriculture.gouv.fr/recensement-agricole- } \\
\text { 2010/resultats-donnees-chiffrees/ }\end{array}$ \\
\hline Guatemala & 2003 & 2 & 419,170 & $\begin{array}{l}\text { Instituto Nacional de Estadística, Guatemala: IV Censo Nacional Agropecuario 2003. Online data: } \\
\underline{\text { http://www.ine.gob.gt/np/agropecuario/tomo\%20IV.pdf }}\end{array}$ \\
\hline Jamaica & 2009 & 1 & 361,540 & Agricultural Business Information System, Jamaica: Livestock Summary Report, 2009 (GLIMS data warehouse) \\
\hline Martinique & 2000 & 1 & 20,621 & $\begin{array}{l}\text { Agreste la Statistique Agricole, Le Ministère de l'Agriculture, de l'Alimentation, de la Pêche et de la Ruralité, } \\
\text { France: Recensement Agricole 2000. Online data: http://agreste.agriculture.gouv.fr/recensement-agricole- } \\
\underline{2010 / \text { resultats-donnees-chiffrees/ }}\end{array}$ \\
\hline Mexico & 2007 & 2 & $8,964,101$ & $\begin{array}{l}\text { Instituto Nacional de Estadística Geografía e Informática, México: Censo Agrícola, Ganadero y Forestal } 2007 . \\
\text { Online data: } \\
\text { http://www.inegi.org.mx/est/contenidos/proyectos/Agro/ca2007/Resultados_Agricola/default.aspx }\end{array}$ \\
\hline Nicaragua & 2001 & 2 & 383,172 & $\begin{array}{l}\text { Instituto Nacional de Estadística y Censos, Nicaragua: III Censo Nacional Agropecuario } 2001 \text { (GLIMS data } \\
\text { warehouse) }\end{array}$ \\
\hline Panama & 2001 & 2 & 312,102 & $\begin{array}{l}\text { Instituto Nacional de Estadística y Censo: Existencia de animales en la República, por clase de animal, según } \\
\text { provincia: } 21 \text { de abril de } 1991 \text { al } 22 \text { abril de 2001. Online data: } \\
\text { http://www.contraloria.gob.pa/inec/Avance/Avance.aspx?ID_CATEGORIA=2\&ID_CIFRAS=8\&ID_IDIO } \\
\underline{\mathrm{MA}=1}\end{array}$ \\
\hline Puerto Rico & 2007 & 2 & 63,585 & $\begin{array}{l}\text { National Agricultural Statistics Service - United States Department of Agriculture: National Agricultural Census } \\
\text { 2007. Online data: http://www.agcensus.usda.gov/Publications/2007/Full_Report/ }\end{array}$ \\
\hline Saint Lucia & 2007 & 6 & 19,520 & $\begin{array}{l}\text { Ministry of Agriculture, Forestry and Fisheries: Census of Agriculture - Final report 2007. Online data: } \\
\underline{\text { http://www.govt.lc/www/publications/AgricultureCensus2007.pdf }}\end{array}$ \\
\hline
\end{tabular}


Saint Vincent and

the Grenadines

United States of

2000

1

America

No Data for: Anguilla, Antigua and Barbuda, Bahamas, Barbados, Bermuda, Bird Island, British V
Netherlands Antilles, Saint Kitts and Nevis, Turks and Caicos Islands, United States Virgin Islands

Netherlands Antilles, Saint Kitts and Nevis, Turks and Caicos Islands, United States Virgin Islands
4,807 Ministry of Agriculture and Labour, Government of Saint Vincent and the Grenadines: National Agricultural Census 2000 (GLIMS data warehouse)

7,239,043 National Agricultural Statistics Service - United States Department of Agriculture: National Agricultural Census 2007. Online data: http://www.agcensus.usda.gov/Publications/2007/Full Report/ 


\section{South America Tile}

\begin{tabular}{|c|c|c|c|c|}
\hline COUNTRY & YEAR & LEVEL & $\begin{array}{l}\text { Totals in } \\
\text { GLIMS }\end{array}$ & SOURCE \\
\hline Argentina & 2002 & 2 & $2,184,445$ & $\begin{array}{l}\text { Instituto Nacional de Estadística y Censos. Argentina: Censo Nacional Agropecuario } 2002 \text { (CNA 2002). Online } \\
\text { data: http://www.indec.gov.ar/agropecuario/cna_principal.asp }\end{array}$ \\
\hline $\begin{array}{l}\text { Bolivia, } \\
\text { Plurinational State } \\
\text { of }\end{array}$ & 2008 & 1 & $2,640,615$ & $\begin{array}{l}\text { Instituto Nacional de Estadística, Bolivia: Encuesta Nacional Agropecuaria. Online data: } \\
\text { http://www.gobernacionlapaz.gob.bo/archivos/Sec_Deptal/SDPD/DID/Estadistica_Deptal/Agropecuario/R } \\
\underline{\text { esultados_ENA_2008/EncuestaNacionalAgropecuaria.pdf }}\end{array}$ \\
\hline Brazil & 2009 & 2 & $38,045,454$ & $\begin{array}{l}\text { Istituto Brasileiro de Geografia e Estatistica: Producao da Pecuria Municipal 2009. Online data: } \\
\text { http://www.ibge.gov.br/home/estatistica/economia/ppm/2009/default.shtm }\end{array}$ \\
\hline Chile & 2007 & 3 & $2,928,606$ & $\begin{array}{l}\text { Instituto Nacional de Estadísticas, Chile: Censo Agropecuario y Forestal 2007. Online data: } \\
\text { http://www.ine.cl/canales/chile_estadistico/censos_agropecuarios/censo_agropecuario_07_comunas.php }\end{array}$ \\
\hline Colombia & 2009 & 1 & $3,908,962$ & Instituto Agropecuario, Colombia: Censo Agropecuario Nacional 2004 - 2009 (GLIMS data warehouse) \\
\hline Ecuador & 2009 & 6 & $1,042,022$ & $\begin{array}{l}\text { Instituto Nacional de Estadística y Censos, Ecuador: Encuesta Nacional de Superficie y Producción Agropecuaria } \\
\text { Continua (ESPAC) (GLIMS data warehouse) }\end{array}$ \\
\hline $\begin{array}{l}\text { Falkland Islands } \\
\text { (Malvinas) }\end{array}$ & 2010 & 1 & 89 & Department of Agriculture, Falkland Islands Government: Statistics Book 2010 (GLIMS data warehouse) \\
\hline French Guiana & 2000 & 6 & 8,087 & $\begin{array}{l}\text { Agreste la Statistique Agricole, Le Ministère de l'Agriculture, de l'Alimentation, de la Pêche et de la Ruralité, } \\
\text { France: Recensement Agricole 2000. Online data: http://agreste.agriculture.gouv.fr/recensement-agricole- } \\
\text { 2010/resultats-donnees-chiffrees/ }\end{array}$ \\
\hline Paraguay & 2008 & 2 & $1,072,655$ & Ministerio de Agricultura y Ganadería, Paraguay: Censo Agropecuario Nacional 2008 (GLIMS data warehouse) \\
\hline Peru & 2006 & 1 & $3,034,134$ & Instituto Nacional de Estadística e Informática, Peru: Compendio Estadístico 2007 (GLIMS data warehouse) \\
\hline Suriname & 1994 & 1 & 42,639 & $\begin{array}{l}\text { Environmental Research Group Oxford, ERGO: ERGO, } 1996 \text { "Livestock Geography. A demonstration of GIS } \\
\text { techniques applied to Global Livestock Systems and Populations", Animal Health Division, FAO, Rome } \\
\text { (William Wint extracted from the agroecological zone estimates derived from the FAOSTAT data for 1994) } \\
\text { (GLIMS data warehouse) }\end{array}$ \\
\hline $\begin{array}{l}\text { Trinidad and } \\
\text { Tobago }\end{array}$ & 2010 & 6 & 35,273 & $\begin{array}{l}\text { Central Statistical Office - Ministry of Planning \& Development Government of the Republic of Trinidad \& } \\
\text { Tobago: Pig Bulletin } 2010 \text { (GLIMS data warehouse) }\end{array}$ \\
\hline Uruguay & 2010 & 1 & 177,914 & $\begin{array}{l}\text { Ministerio de Ganadería, Agricultura y Pesca, Estadísticas Agropecuarias, Uruguay: Datos de la Declaración } \\
\text { Jurada de DICOSE } 2010 \text { - Datos Generales de Lechería y de Suinos (GLIMS data warehouse) }\end{array}$ \\
\hline $\begin{array}{l}\text { Venezuela, } \\
\text { Bolivarian Republic }\end{array}$ & 2007 & 2 & $2,787,338$ & $\begin{array}{l}\text { Ministerio del Poder Popular para la Agricultura y Tierras - Venezuela: VII Censo Agrícola 2007. Online data: } \\
\text { http://censo.mat.gob.ve/ }\end{array}$ \\
\hline
\end{tabular}




\section{Oceania Tile}

\begin{tabular}{|c|c|c|c|c|}
\hline COUNTRY & YEAR & LEVEL & $\begin{array}{l}\text { Totals in } \\
\text { GLIMS }\end{array}$ & SOURCE \\
\hline American Samoa & 2008 & 1 & 16,904 & $\begin{array}{l}\text { National Agricultural Statistics Service - United States Department of Agriculture: National Agricultural Census } \\
\text { 2007. Online data: http://www.agcensus.usda.gov/Publications/2007/Full_Report/ }\end{array}$ \\
\hline Australia & 2007 & 6 & $2,469,334$ & $\begin{array}{l}\text { Australian Bureau of Statistics: Agricultural commodities: small area data, Australia 2006-2007 (GLIMS data } \\
\text { warehouse) }\end{array}$ \\
\hline New Caledonia & 2002 & 2 & 25,447 & $\begin{array}{l}\text { Institut de la Statistique et des Etudes Economiques (ISEE): Recensement Général Agricole (RGA) 2002. Online } \\
\text { data: } \\
\text { http://www.davar.gouv.nc/portal/page/portal/davar/secteur_rural/statistiques_agricoles/donnees_synthese/re } \\
\text { censement_agricole }\end{array}$ \\
\hline New Zealand & 2010 & 1 & 302,073 & $\begin{array}{l}\text { Statistics New Zealand: } 2011 \text { livestock numbers by Regional council. Online data: } \\
\text { http://www.stats.govt.nz/tools_and_services/nzdotstat/agriculture-statistics.aspx }\end{array}$ \\
\hline $\begin{array}{l}\text { Northern Mariana } \\
\text { Islands }\end{array}$ & 2007 & 1 & 2,242 & $\begin{array}{l}\text { National Agricultural Statistics Service - United States Department of Agriculture: National Agricultural Census } \\
\text { 2007. Online data: http://www.agcensus.usda.gov/Publications/2007/Full_Report/ }\end{array}$ \\
\hline Tonga & 2001 & 1 & 113,580 & $\begin{array}{l}\text { Ministry of Agriculture and Forestry, Kingdom of Tonga: Agriculture Census 2001. Online data: } \\
\text { http://www.spc.int/prism/tonga/index.php/surveys/agriculture-census-2011 }\end{array}$ \\
\hline
\end{tabular}

作,

Guinea, Pitcairn, Samoa, Solomon Islands, Tokelau, Tuvalu, Vanuatu, Wake Island, Wallis and Futuna 
Supplementary information $5 \mathrm{c}$ - Country details on sub-national statistics for chickens (year and level of observation) by continental tile.

\section{Africa Tile}

\begin{tabular}{|c|c|c|c|c|}
\hline COUNTRY & YEAR & LEVEL & Totals in GLIMS & SOURCE \\
\hline Angola & 2010 & 1 & $17,118,618$ & $\begin{array}{l}\text { CountrySTAT - Food and agriculture data network: Número de animais vivos by Ano, Especie, Nivel } \\
\text { administrativo 1. Online data: http:// countrystat.org/home.aspx?c=AGO\&ta=007SPD035\&tr=21 }\end{array}$ \\
\hline Benin & 2009 & 2 & $15,286,300$ & $\begin{array}{l}\text { CountrySTAT - Food and agriculture data network: Élevage - Total des effectifs d'animaux vivants } 2010 \text { - } \\
\text { 2012. Online data: http://www.countrystat.org/home.aspx?c=BEN\&ta=053SPD135\&tr=21 }\end{array}$ \\
\hline Botswana & 2006 & 1 & $1,639,141$ & $\begin{array}{l}\text { Central Statistics Office Republic of Botswana: Botswana Agricultural Census Report. Ministry of Finance } \\
\text { and Development Planning, } 2007 \text { (GLIMS data warehouse) }\end{array}$ \\
\hline Burkina Faso & 2008 & 2 & $28,267,052$ & $\begin{array}{l}\text { Ministère des Ressources Animales: Les Statistiques du Secteur de l’Élevage au Burkina Faso, } 2003 \text { \& } 2008 \\
\text { (GLIMS data warehouse) }\end{array}$ \\
\hline Burundi & 2007 & 1 & $1,267,675$ & $\begin{array}{l}\text { CountrySTAT - Food and agriculture data network: Élevage - Total des effectifs d'animaux vivants. . } \\
\text { Online data: } \underline{\text { http://www.countrystat.org/home.aspx?c=BDI\&ta=029SPD035\&tr=21 }}\end{array}$ \\
\hline Côte d'Ivoire & 2001 & 1 & $17,125,712$ & $\begin{array}{l}\text { Ministère de l'Agriculture et des Ressources Animales, Direction de la Programmation, Côte d'Ivoire: } \\
\text { Recensement National de l'Agriculture } 2001 \text { (GLIMS data warehouse) }\end{array}$ \\
\hline Cameroon & 2007 & 2 & $11,411,072$ & CountrySTAT - Food and agriculture data network (GLIMS data warehouse) \\
\hline Cape Verde & 2004 & 2 & 327,562 & Directorate of Livestock, Cape Verde: Recenseamento Geral da Agricultura, 2004 (GLIMS data warehouse) \\
\hline $\begin{array}{l}\text { Central African } \\
\text { Republic }\end{array}$ & 1990 & 1 & $2,773,110$ & $\begin{array}{l}\text { Environmental Research Group Oxford, ERGO: FAO, 2004. Agro-ecological distributions for Africa } \\
\text { (GLIMS data warehouse) }\end{array}$ \\
\hline Chad & 2009 & 1 & $47,867,370$ & Ministère de l'Élevage et des Ressources Animales: Revue du secteur avicole (GLIMS data warehouse) \\
\hline Comoros & 2000 & 1 & 221,550 & $\begin{array}{l}\text { FAO Animal Production and Health Division: Rapport National Sur l'Etat des Ressources Génétiques } \\
\text { Animales (GLIMS data warehouse) }\end{array}$ \\
\hline $\begin{array}{l}\text { Democratic } \\
\text { Republic of the } \\
\text { Congo }\end{array}$ & 1994 & 1 & $20,561,809$ & Services national des Statistiques Agricoles 1994 (GLIMS data warehouse) \\
\hline Egypt & 2005 & 1 & $68,452,380$ & $\begin{array}{l}\text { Economic Affaire Sector, Ministry of Agriculture and Land Reclamation, Egypt: Poultry in the } 21 \text { st century } \\
\text { (GLIMS data warehouse) }\end{array}$ \\
\hline Ethiopia & 2010 & 2 & $49,286,935$ & Central Statistical Authority, Ethiopia: Agricultural Sample Survey 2008 and 2010 (GLIMS data warehouse) \\
\hline Gabon & 2002 & 1 & $3,100,001$ & $\begin{array}{l}\text { Environmental Research Group Oxford, ERGO: FAO, 2004. Agro-ecological distributions for Africa } \\
\text { (GLIMS data warehouse) }\end{array}$ \\
\hline
\end{tabular}




$\begin{array}{lll}\text { Gambia } & 2007 & 1 \\ \text { Ghana } & 2009 & 1 \\ \text { Guinea } & 2002 & 2 \\ \text { Guinea-Bissau } & 1994 & 2 \\ \text { Kenya } & 2009 & 2 \\ \text { Lesotho } & 2008 & 1 \\ \text { Madagascar } & 2005 & 3 \\ \text { Malawi } & 2007 & 2 \\ \text { Mali } & 2010 & 1 \\ \text { Mozambique } & 2002 & 2 \\ \text { Namibia } & 2001 & 1 \\ \text { Niger } & 2007 & 1 \\ & & 1 \\ \text { Nigeria } & 2006 & 1 \\ \text { Rwanda } & 2008 & 1 \\ \text { Réunion } & 2000 & 1 \\ \text { Saint Helena } & 2009 & 1 \\ \text { Sao Tome and } & 1997 & \\ \text { Principe } & & \\ \text { Senegal } & 2009 & \\ \end{array}$

820,438 National Agriculture Development Agency, Gambia: National Agricultural Sample Survey Report (NASS), Oct. 2008 (GLIMS data warehouse)

33,140,820 CountrySTAT - Food and agriculture data network (GLIMS data warehouse)

13,213,984 Service National des Statistiques Agricoles, République de Guinée 2002 (GLIMS data warehouse)

729,029 Instituto Nacional de Estadística: Inquérito Anual sobre Superficie Rendimento e Producao. Campanha 93/94 (GLIMS data warehouse)

32,217,042 Kenya National Bureau of Statistics: Kenyan Livestock Population 2009 (GLIMS data warehouse)

715,576 Bureau of Statistics of Lesotho: Livestock winter 2008/09 Tables. Online data: http://www.bos.gov.ls/Downloads.htm

24,213,520 Ministère de l'Agriculture, Secrétariat General, Direction de la Prévision et des Etudes Economiques, Madagascar: Recensement de l'agriculture (RA) - Campagne agricole 2004-2005 (GLIMS data warehouse)

7,557,745 Agriculture Statistics Division of the National Statistical Office of Malawi (NSO): National Census of Agriculture and Livestock (October 2006 - October 2007) (GLIMS data warehouse)

36,750,000 CountrySTAT - Food and agriculture data network: Élevage - Total des effectifs d'animaux vivants. Online data: http://www.countrystat.org/home.aspx?c=MLI\&tr=21

22,636,314 Direccao Nacional de Pecuaria, Mozambique: Annual Reports, 2003 (GLIMS data warehouse)

458,010 Department of Veterinary Services, Namibia: FAO Special Report, FAO/WFP Crop, Livestock and Food Security Assessment Mission to Namibia (GLIMS data warehouse)

6,674,216 Ministère des Ressources Animales: Recensement Général de l'Agriculture et du Cheptel (RGAC

2005/2007). Online data: http://harvestchoice.org/publications/niger-recensement-general-de-lagricultureet-du-cheptel-rgac-20052007-volume-v-producti

2

52,383,612 National Bureau of Statistics, Federal Ministry of Agriculture and Rural Development: Estimated Subsistence Poultry Population by State (2006 estimates) (GLIMS data warehouse)

2,894,972 Ministère de l'Agriculture et de l'élevage: FAO, Revue du secteur agricole: RWANDA 2008 (GLIMS data warehouse)

1,277,621 Agreste la Statistique Agricole, Le Ministère de l'Agriculture, de l'Alimentation, de la Pêche et de la Ruralité, France: Recensement Agricole 2000. Online data: http://agriculture.gouv.fr/recensement-agricole-2010

4,421 Agriculture and Natural Resources Department of St. Helena: Livestock Census 2009 (GLIMS data warehouse)

37,608 Ministère de l'Agriculture et de la Pêche: Enquête Agricole de Production, EAP 1997 (GLIMS data warehouse)

22,301,806 Agence Nationale de la Statistique et de la Démographie du Sénégal: Live Data Base (LDB). Online data: http://www.ansd.sn/publications_SES_region.html 
Sierra Leone

2004

2002

2002

2005

2010

2000

Uganda

United Republic of
3,227,252 Ministry of Agriculture \& Food Security of Sierra Leone: 2004 Populations and Housing Census Statistical Office (GLIMS data warehouse)

3,299,999 Environmental Research Group Oxford, ERGO: Agro-ecological distributions for Africa (GLIMS data warehouse)

45,400,346 Directorate Veterinary Services, South Africa: Animal Disease Report, January to September 2003 (GLIMS data warehouse)

35,024,922 Ministry of Animal Resources: Estimates supplied by veterinary staff at Ministry of Animal Resources for 2005 (GLIMS data warehouse)

1,936,344 Directorate of Veterinary and Livestock Services: Swaziland Livestock Statistics, Census 2010 (GLIMS data warehouse)

12,759,176 Uganda Bureau of Statistics: Uganda census 2000 - Data acquired from W. Wint (GLIMS data warehouse)

26,839,376 CountrySTAT - Food and agriculture data network (GLIMS data warehouse)

10,421,085 Department of Research and Specialist Services, Zambia: National Livestock Census by Province 1994 to 2009 (GLIMS data warehouse)

No Data for: Algeria, Bassas da India, British Indian Ocean Territory, Congo, Djibouti, Equatorial Guinea, Eritrea, Europa Island, Glorioso Island, Juan de Nova Island, Liberia, Libya, Mauritania,

Mauritius, Mayotte, Morocco, Seychelles, Togo, Tromelin Island, Tunisia, Western Sahara, Zimbabwe 


\section{Asia Tile}

\begin{tabular}{|c|c|c|c|c|}
\hline COUNTRY & YEAR & LEVEL & Totals in GLIMS & SOURCE \\
\hline Afghanistan & 2003 & 2 & $12,136,388$ & $\begin{array}{l}\text { Ministry of Agriculture, Irrigation and Livestock of Afghanistan: Afghanistan - National Livestock Census } \\
\text { 2002-2003 - Final Report. Online data: } \\
\text { http://reliefweb.int/sites/reliefweb.int/files/resources/58C374339FC582F149256DF30009D60F-fao-afg- } \\
\text { 4dec.pdf }\end{array}$ \\
\hline Bangladesh & 2005 & 6 & $197,456,543$ & $\begin{array}{l}\text { Bangladesh Bureau of Statistics: Data Retrieved } 12 \text { November } 2009 \text { from http://www.bbs.gov.bd/ } \\
\text { (GLIMS data warehouse) }\end{array}$ \\
\hline Belarus & 2011 & 1 & $36,410,987$ & $\begin{array}{l}\text { National Statistical Committee of the Republic of Belarus: Agriculture of the Republic of Belarus, } 2011 \\
\text { (GLIMS data warehouse) }\end{array}$ \\
\hline Bhutan & 2008 & 1 & 198,148 & National Statistics Bureau, Bhutan: Annual district statistics 2010 (GLIMS data warehouse) \\
\hline Cambodia & 2009 & 2 & $15,103,537$ & $\begin{array}{l}\text { Department of Animal Production and Health, Cambodia: } 2009 \text { district data (David Bourn, } \\
\text { "Environmental Animal Health Management Initiative for Enhanced Smallholder Production", 2010) } \\
\text { (GLIMS data warehouse) }\end{array}$ \\
\hline China & 2005 & 6 & $5,012,700,208$ & $\begin{array}{l}\text { Editorial Department of China Animal Husbandry: China animal husbandry yearbook. Beijing, China: } \\
\text { China Agricultural Press (GLIMS data warehouse) }\end{array}$ \\
\hline Georgia & 2004 & 2 & $7,960,916$ & $\begin{array}{l}\text { National Statistics Office of Georgia: First National Agricultural Census } 2004 \text { in Georgia. Online data: } \\
\text { http://geostat.ge/cms/site_images/_files/english/agriculture/census/section7.pdf }\end{array}$ \\
\hline India & 2007 & 2 & $606,978,885$ & $\begin{array}{l}\text { Department of Animal Husbandry, Dairying \& Fisheries, Ministry of Agriculture, India: Census of } \\
\text { Agriculture, } 2007 \text { (GLIMS data warehouse) }\end{array}$ \\
\hline Indonesia & 2010 & 1 & $1,349,625,877$ & Department Pertanian Republik Indonesia: Buku Statistik, 2011 (GLIMS data warehouse) \\
\hline Japan & 2009 & 1 & $285,349,000$ & $\begin{array}{l}\text { Statistics Department, Minister's Secretariat, Ministry of Agriculture, Forestry and Fisheries: Japan } \\
\text { Statistical Yearbook 2010. Online data: } \\
\text { http://www.maff.go.jp/e/tokei/kikaku/nenji_e/85nenji/index.html }\end{array}$ \\
\hline Jordan & 2007 & 1 & 172,052 & Department of Statistics, Jordan: The Agricultural Census 2007 (GLIMS data warehouse) \\
\hline Kazakhstan & 2004 & 1 & $25,480,500$ & $\begin{array}{l}\text { Agency on Statistics of the Republic of Kazakhstan: Main socio economic indicators of regions in } \\
\text { Kazakhstan 2005. Almaty, Kazakhstan (GLIMS data warehouse) }\end{array}$ \\
\hline Kuwait & 2000 & 1 & $26,314,863$ & $\begin{array}{l}\text { Central Statistical Office and Public Authority for Agriculture and Fisheries: Annual Statistical Abstract } \\
2000 \text { (GLIMS data warehouse) }\end{array}$ \\
\hline Kyrgyzstan & 2003 & 1 & $3,585,842$ & $\begin{array}{l}\text { National Statistic Committee of Kyrgyzstan: Results of the first agricultural census of the Kyrgyz Republic } \\
\text { of } 2003 \text { (Second Stage) (GLIMS data warehouse) }\end{array}$ \\
\hline
\end{tabular}


Malaysia

Myanmar

Nepal

Pakistan

Philippines

Republic of Korea

Romania

Russian Federation

Saudi Arabia

Sri Lanka

Syrian Arab

Republic

Tajikistan

Thailand

Turkey

Ukraine
$16,047,773$

Department of Livestock and Fisheries, Laos: 2009 district data (David Bourn, “Environmental Animal Health Management Initiative for Enhanced Smallholder Production”, 2010) (GLIMS data warehouse)

12,053,000 United Nations, Economic and Social Commission for Western Asia, 1999: National farm data handbook for Lebanon. New York (GLIMS data warehouse)

119,980,226 Department of Veterinary Service, Malaysia: Livestock Products statistics 1996-2002 (GLIMS data warehouse)

82,154,065 Livestock Breeding and Veterinary Department (LBVD), Ministry of Livestock Breeding and Fisheries, Yangon, Myanmar: Myanmar Animal Census 2006 (GLIMS data warehouse)

24,333,826 Ministry of Agriculture and Cooperatives (MOAC), Nepal: 2009 data (GLIMS data warehouse)

59,253,879 Agricultural Census Organization, Ministry of Food \& Agriculture, Pakistan: Livestock census 1996 special report. Lahore, Pakistan (GLIMS data warehouse)

135,912,594 Bureau of Animal Industry, Philippines: 2006 data (David Bourn, “ Environmental Animal Health Management Initiative for Enhanced Smallholder Production”, 2010) (GLIMS data warehouse)

109,627,646 Korea National Statistical Office: Korea Statistical Yearbook 2005 (GLIMS data warehouse)

69,583,725 EUROSTAT on line database. Section: General Statistics, Region, Agriculture, Animal Populations and Land Use. February 2005. Online data:

http://epp.eurostat.ec.europa.eu/portal/page/portal/agriculture/data/

216,620,630 Russian Federation - Federal State Statistical Service: Results of All-Russia Agricultural Census 2006 (1-8 vol.) (GLIMS data warehouse)

531,875,811 FAO Animal Production and Health Division: Saudi Arabia Poultry Sector Country Review (GLIMS data warehouse)

15,617,153 Agriculture and Environment Statistics Division, Department of Census and Statistics, Sri Lanka: Census of Agriculture 2002, All Sectors. Online data: http://www.statistics.gov.lk/agriculture/AGC2002/AGC2002.htm

28,502,000 FAO Animal Production and Health Division, Emergency Centre for Transboundary Animal Diseases, Socio Economics, Production and Biodiversity Unit, 2008. Poultry sector country review: Syria (GLIMS data warehouse)

1,061,500 Government Statistic Committee of the Republic of Tajikistan: Agricultural Yearbook of the Republic of Tajikistan (GLIMS data warehouse)

191,375,293 Department of Livestock Development, Ministry of Agriculture and Cooperatives, Thailand: Census 2004 (X- ray surveys searching for HPAI outbreaks) (GLIMS data warehouse)

230,105,873 Turkish Statistical Institute: Livestock statistics database 2009 (GLIMS data warehouse)

159,218,996 State Statistics Committee Ukraine: Head of livestock and poultry by regions (GLIMS data warehouse) 
148,078,344 General Statistics Office of Vietnam: Agricultural Census database 2001. Online data: http://www.gso.gov.vn/default_en.aspx?tabid=477\&idmid=4\&ItemID=1824

No Data for: Armenia, Azerbaijan, Bahrain, Brunei Darussalam, Bulgaria, Christmas Island, Cocos (Keeling) Islands, Cyprus, Democratic People’s Republic of Korea, Islamic Republic of Iran, Iraq, Israel, Kuril Islands, Liancourt Rock, Maldives, Mongolia, Oman, Paracel Islands, Qatar, Republic of Moldova, Scarborough Reef, Senkaku Islands, Singapore, Spratly Islands, Timor-Leste,

Turkmenistan, United Arab Emirates, Uzbekistan, Yemen 


\section{Europe Tile}

\begin{tabular}{|c|c|c|c|c|}
\hline COUNTRY & YEAR & LEVEL & Totals in GLIMS & SOURCE \\
\hline Albania & 2010 & 1 & $5,244,500$ & Institute of Statics, Albania: Agricultural sector, Livestock 2001-2010 (GLIMS data warehouse) \\
\hline Austria & 2003 & 1 & $12,571,600$ & $\begin{array}{l}\text { EUROSTAT online database. General Statistics, Region, Agriculture, Animal Populations and Land Use. } \\
\text { Online data: http://epp.eurostat.ec.europa.eu/portal/page/portal/agriculture/data/ }\end{array}$ \\
\hline Belgium & 2003 & 2 & $32,032,000$ & $\begin{array}{l}\text { EUROSTAT online database. General Statistics, Region, Agriculture, Animal Populations and Land Use. } \\
\text { Online data: http://epp.eurostat.ec.europa.eu/portal/page/portal/agriculture/data/ }\end{array}$ \\
\hline Croatia & 2003 & 1 & $10,477,514$ & $\begin{array}{l}\text { Central Bureau of Statistics, Republic of Croatia: Agricultural Census 2003. Online data: } \\
\underline{\text { http://www.dzs.hr/default_e.htm }}\end{array}$ \\
\hline Czech Republic & 2010 & 2 & $16,843,696$ & $\begin{array}{l}\text { Czech Statistical Office: Agrocensus regions: Farm Structure Survey and Survey on Agricultural Production } \\
\text { Methods 2010. Online data: http://www.czso.cz/csu/2012edicniplan.nsf/engp/2127-12 }\end{array}$ \\
\hline Denmark & 2010 & 2 & $18,083,961$ & $\begin{array}{l}\text { Denmark Statistics: Farms by region and selected farms, crops and livestock. Online data: } \\
\underline{\text { http://www.statistikbanken.dk/statbank5a/SelectVarVal/Define.asp?Maintable=BDF51\&PLanguage=1 }}\end{array}$ \\
\hline Estonia & 2001 & 2 & $1,611,794$ & $\begin{array}{l}\text { Statistikaamet Statistical Office of Estonia: Agricultural Census 2001. Online data: } \\
\text { http://www.stat.ee/agricultural-census-2001 }\end{array}$ \\
\hline Finland & 2010 & 6 & $7,828,600$ & $\begin{array}{l}\text { The Information Centre of the Ministry of Agriculture and Forestry, Finland: Yearbook of Farm Statistics } \\
2011 \text { (GLIMS data warehouse) }\end{array}$ \\
\hline France & 2001 & 2 & $205,617,000$ & $\begin{array}{l}\text { Agreste la Statistique Agricole, Le Ministère de l'Agriculture, de l'Alimentation, de la Pêche et de la Ruralité: } \\
\text { Agreste la Statistique Agricole, } 2002 \text { (GLIMS data warehouse) }\end{array}$ \\
\hline Germany & 2001 & 1 & $109,993,100$ & $\begin{array}{l}\text { Federal Statistical Office, Germany: Statistical Yearbook } 2003 \text { for the Federal Republic of Germany } \\
\text { (GLIMS data warehouse) }\end{array}$ \\
\hline Greece & 2007 & 2 & $33,495,026$ & $\begin{array}{l}\text { Hellenic Statistic Authority (EL.STAT): Farms and number of animals by type, region and county (GLIMS } \\
\text { data warehouse) }\end{array}$ \\
\hline Hungary & 2010 & 1 & $31,849,000$ & Hungarian Central Statistical Office: Regional Statistics. Online data: http://www.ksh.hu/agriculture \\
\hline Iceland & 2010 & 2 & 323,427 & Statistics Iceland: 2010 data (GLIMS data warehouse) \\
\hline Ireland & 2000 & 1 & $12,724,881$ & $\begin{array}{l}\text { Central Statistical Office Ireland: Census of Agriculture 2000. Online data: } \\
\underline{\text { http://www.cso.ie/en/releasesandpublications/agricultureandfishing/censusofagriculturemainresultsjune20 }} \\
\underline{00 /}\end{array}$ \\
\hline Italy & 2010 & 3 & $139,031,114$ & $\begin{array}{l}\text { Istituto Nazionale di Statistica: Censimento Agricoltura 2010. Online data: http://dati- } \\
\text { censimentoagricoltura.istat.it }\end{array}$ \\
\hline Latvia & 2001 & 1 & $3,470,101$ & $\begin{array}{l}\text { Central Statistical Bureau of Latvia: Results of } 2001 \text { Agricultural Census. Online data: } \\
\text { http://www.csb.gov.lv/en/dati/agricultural-census-2001-30765.html }\end{array}$ \\
\hline Liechtenstein & 2009 & 6 & 12,000 & Amt für Statistik Liechtenstein: Landwirtschaftsstatistik 2009 (GLIMS data warehouse) \\
\hline
\end{tabular}




\begin{tabular}{|c|c|}
\hline Lithuania & 2010 \\
\hline Luxembourg & 2008 \\
\hline Malta & 2010 \\
\hline Montenegro & 2010 \\
\hline Netherlands & 2010 \\
\hline Norway & 2002 \\
\hline Poland & 2009 \\
\hline Portugal & 2009 \\
\hline Serbia & 2006 \\
\hline Slovakia & 2009 \\
\hline Slovenia & 2010 \\
\hline Spain & 2009 \\
\hline Sweden & 2009 \\
\hline Switzerland & 2009 \\
\hline The former & 2008 \\
\hline $\begin{array}{l}\text { Yugoslav Republic } \\
\text { of Macedonia }\end{array}$ & \\
\hline United Kingdom & 2009 \\
\hline
\end{tabular}

9,465,819 Lietuvos Statistikas Departamenta: Number of livestock by administrative territory, 2011 (GLIMS data warehouse)

100,787 Portail des statistiques du Grand-Duché de Luxembourg (STATEC): Recensement Agricole, 2008 (GLIMS data warehouse)

1,146,810 National Statistics Office of Malta: Census of Agriculture 2010. Online data: http://www.nso.gov.mt/statdoc/document_view.aspx?id=3287\&backurl=/themes/theme_page.aspx

411,083 Statistical office of Montenegro (MONSTAT): Agriculture Census 2010. Online data: http://www.monstat.org/eng/page.php?id $=58$ \&pageid $=58$

101,247,711 Centraal Bureau voor de Statistiek, Netherlands: Agriculture: crops, livestock and land use by general far type, region - 2011 (GLIMS data warehouse)

3,137,661 Statistics Norway: Statistical Yearbook 2003 (GLIMS data warehouse)

124,129,100 Central Statistical Office, Poland: Statistical Yearbook of Agriculture 2010 (GLIMS data warehouse)

34,368,861 Instituto Nacional de Estatística - Statistics Portugal: Agricultural Census 2009. Online data: http://ra09.ine.pt/xportal/xmain?xpid=RA2009\&xpgid=ra_home

16,595,204 Statistical Office of Serbia: Municipalities of Serbia 2007 (GLIMS data warehouse)

13,104,090 Statistical Office of the Slovak Republic: Livestock by territory, products and period. Online data: http://px-web.statistics.sk/PXWebSlovak/DATABASE/En/databasetree.asp

4,005,852 Statistical Office of the Republic of Slovenia: Agricultural Census Slovenia 2010. Online data: http://www.stat.si/eng/novica_prikazi.aspx?id=4594

178,328,136 Instituto Nacional de Estadística, Spain: Agrarian Census 2009. Online data: http:/ $/$ www.ine.es $/$ jaxi $/$ menu.do?type $=$ pcaxis\&path $=\% 2 \mathrm{Ft} 01 \% 2 \mathrm{Fp} 042 / \mathrm{E} 01 \&$ file $=$ inebase $\& \mathrm{~L}=1$

7,158,272 Statistics Sweden: Yearbook of Agricultural Statistics 2010 (GLIMS data warehouse)

8,741,117 Swiss Federal Statistical Office: Recensement des entreprises agricoles,1999-2009 (GLIMS data warehouse)

2,226,055 State Statistic Office of the Republic of Macedonia: Total number of livestock, poultry and bee-hives (2007 and 2008) (GLIMS data warehouse)

131,354,251 Office for National Statistics, U.K.: Statistics by Region. Online data: http://www.statistics.gov.uk/hub/regional-statistics/

No Data for: Andorra, Bosnia and Herzegovina, Faroe Islands, Gibraltar, Guernsey, Holy See, Isle of Man, Jersey, Madeira Islands, Monaco, San Marino, Svalbard and Jan Mayen Islands 


\section{North America Tile}

\begin{tabular}{|c|c|c|c|c|}
\hline COUNTRY & YEAR & LEVEL & Totals in GLIMS & SOURCE \\
\hline Belize & 2002 & 1 & $1,764,435$ & $\begin{array}{l}\text { Ministry Of Agriculture, Fisheries and Cooperatives, Belize: Annual Report 2002. Online data: } \\
\text { http://www.agriculture.gov.bz/PDF/2002_annual_report.pdf }\end{array}$ \\
\hline Canada & 2006 & 2 & $118,641,120$ & $\begin{array}{l}\text { Statistics Canada: } 2006 \text { Census of Agriculture. Online data: http://www.statcan.gc.ca/ca-ra2006/index- } \\
\text { eng.htm }\end{array}$ \\
\hline El Salvador & 2007 & 2 & $35,123,656$ & Ministerio de Economía - El Salvador: IV Censo Agropecuario 2007-2008 (GLIMS data warehouse) \\
\hline Guatemala & 2003 & 2 & $21,518,212$ & $\begin{array}{l}\text { Instituto Nacional de Estadística, Guatemala: IV Censo Nacional Agropecuario 2003. Online data: } \\
\text { http://www.ine.gob.gt/np/agropecuario/tomo\%20IV.pdf }\end{array}$ \\
\hline Jamaica & 2009 & 1 & $11,026,836$ & $\begin{array}{l}\text { Agricultural Business Information System, Jamaica: Livestock Summary Report, } 2009 \text { (GLIMS data } \\
\text { warehouse) }\end{array}$ \\
\hline Martinique & 2000 & 1 & 407,811 & $\begin{array}{l}\text { Agreste la Statistique Agricole, Le Ministère de l'Agriculture, de l'Alimentation, de la Pêche et de la Ruralité, } \\
\text { France: Recensement Agricole 2000. Online data: http://agreste.agriculture.gouv.fr/recensement-agricole- } \\
\text { 2010/resultats-donnees-chiffrees/ }\end{array}$ \\
\hline Mexico & 2007 & 2 & $154,126,180$ & $\begin{array}{l}\text { Instituto Nacional de Estadística Geografía e Informática, México: Censo Agrícola, Ganadero y Forestal } \\
\text { 2007. Online data: } \\
\text { http://www.inegi.org.mx/est/contenidos/proyectos/Agro/ca2007/Resultados_Agricola/default.aspx }\end{array}$ \\
\hline Nicaragua & 2001 & 2 & $8,422,682$ & $\begin{array}{l}\text { Instituto Nacional de Estadística y Censos, Nicaragua: III Censo Nacional Agropecuario } 2001 \text { (GLIMS data } \\
\text { warehouse) }\end{array}$ \\
\hline Panama & 2001 & 2 & $14,082,185$ & $\begin{array}{l}\text { Instituto Nacional de Estadística y Censo: Existencia de Animales en la República, por clase de animal, } \\
\text { según provincia: } 21 \text { de abril de } 1991 \text { al } 22 \text { abril de 2001. Online data: } \\
\text { http://www.contraloria.gob.pa/inec/Avance/Avance.aspx?ID_CATEGORIA=2\&ID_CIFRAS=8\&ID_I } \\
\underline{\text { DIOMA=1 }}\end{array}$ \\
\hline Puerto Rico & 2007 & 2 & $5,031,810$ & $\begin{array}{l}\text { National Agricultural Statistics Service - United States Department of Agriculture: National Agricultural } \\
\text { Census 2007. Online data: http://www.agcensus.usda.gov/Publications/2007/Full_Report/ }\end{array}$ \\
\hline Saint Lucia & 2007 & 6 & 129,861 & $\begin{array}{l}\text { Ministry of Agriculture, Forestry and Fisheries: Census of Agriculture - Final report 2007. Online data: } \\
\text { http://www.govt.lc/www/publications/AgricultureCensus2007.pdf }\end{array}$ \\
\hline $\begin{array}{l}\text { Saint Vincent and } \\
\text { the Grenadines }\end{array}$ & 2000 & 1 & 65,920 & $\begin{array}{l}\text { Ministry of Agriculture and Labour, Government of Saint Vincent and the Grenadines: National } \\
\text { Agricultural Census } 2000 \text { (GLIMS data warehouse) }\end{array}$ \\
\hline $\begin{array}{l}\text { United States of } \\
\text { America }\end{array}$ & 2007 & 2 & $1,692,431,565$ & $\begin{array}{l}\text { National Agricultural Statistics Service - United States Department of Agriculture: National Agricultural } \\
\text { Census 2007. Online data: http://www.agcensus.usda.gov/Publications/2007/Full Report/ }\end{array}$ \\
\hline
\end{tabular}




\section{South America Tile}

\begin{tabular}{|c|c|c|c|c|}
\hline COUNTRY & YEAR & LEVEL & Totals in GLIMS & SOURCE \\
\hline Argentina & 2002 & 2 & $244,131,110$ & $\begin{array}{l}\text { Instituto Nacional de Estadística y Censos. Argentina: Censo Nacional Agropecuario } 2002 \text { (CNA 2002). } \\
\text { Online data: http://www.indec.gov.ar/agropecuario/cna_principal.asp }\end{array}$ \\
\hline $\begin{array}{l}\text { Bolivia, } \\
\text { Plurinational State } \\
\text { of }\end{array}$ & 2009 & 1 & $161,268,733$ & $\begin{array}{l}\text { Instituto Nacional de Estadística, Bolivia: Existencia Total de Aves Parrilleras por Ano, Según } \\
\text { Departamento 2000-2009 (GLIMS data warehouse) }\end{array}$ \\
\hline Brazil & 2009 & 2 & $1,024,999,717$ & $\begin{array}{l}\text { Istituto Brasileiro de Geografia e Estatistica: Producao da Pecuria Municipal 2009. Online data: } \\
\text { http://www.ibge.gov.br/home/estatistica/economia/ppm/2009/default.shtm }\end{array}$ \\
\hline Chile & 2007 & 1 & $40,362,000$ & $\begin{array}{l}\text { Instituto Nacional de Estadísticas, Chile: Censo Agropecuario y Forestal 2007. Online data: } \\
\text { http://www.ine.cl/canales/chile_estadistico/censos_agropecuarios/censo_agropecuario_07_comunas.php }\end{array}$ \\
\hline Colombia & 2009 & 1 & $144,913,543$ & Instituto Agropecuario, Colombia: Censo Agropecuario Nacional 2004 - 2009 (GLIMS data warehouse) \\
\hline Ecuador & 2000 & 2 & $40,823,593$ & $\begin{array}{l}\text { Project SICA, Agricultural Information System, Ministry of Agriculture and Livestock, Ecuador: III Censo } \\
\text { Nacional Agropecuario (GLIMS data warehouse) }\end{array}$ \\
\hline French Guiana & 1994 & 6 & 398,988 & $\begin{array}{l}\text { Institut National de la Statistique et des Etudes Economiques (INSEE): Tableaux Economiques Régionaux. } \\
\text { Guyane 1995. Online data: } \underline{\text { http://www.insee.fr/fr/regions/guyane/collection.asp?id=76 }}\end{array}$ \\
\hline Paraguay & 2008 & 2 & $16,055,854$ & $\begin{array}{l}\text { Ministerio de Agricultura y Ganadería, Paraguay: Censo Agropecuario Nacional } 2008 \text { (GLIMS data } \\
\text { warehouse) }\end{array}$ \\
\hline Peru & 2007 & 1 & $120,226,000$ & $\begin{array}{l}\text { Instituto Nacional de Estadística e Informática, Peru: Compendio Estadístico } 2007 \text { (GLIMS data } \\
\text { warehouse) }\end{array}$ \\
\hline Suriname & 1994 & 1 & $6,459,453$ & $\begin{array}{l}\text { Environmental Research Group Oxford, ERGO: ERGO, } 1996 \text { "Livestock Geography. A demonstration of } \\
\text { GIS techniques applied to Global Livestock Systems and Populations", Animal Health Division, FAO, } \\
\text { Rome (William Wint extracted from the agroecological zone estimates derived from the FAOSTAT data for } \\
\text { 1994) (GLIMS data warehouse) }\end{array}$ \\
\hline Uruguay & 2000 & 1 & $9,912,543$ & $\begin{array}{l}\text { Instituto Nacional de Estadística, Republica Oriental del Uruguay: Censo Agropecuario } 2000 \text { (GLIMS data } \\
\text { warehouse) }\end{array}$ \\
\hline $\begin{array}{l}\text { Venezuela, } \\
\text { Bolivarian Republic }\end{array}$ & 2007 & 1 & $91,417,167$ & $\begin{array}{l}\text { Ministerio del Poder Popular para la Agricultura y Tierras - Venezuela: VII Censo Agrícola 2007. Online } \\
\text { data: http://censo.mat.gob.ve/ }\end{array}$ \\
\hline
\end{tabular}




\section{Oceania Tile}

\begin{tabular}{|c|c|c|c|c|}
\hline COUNTRY & YEAR & LEVEL & Totals in GLIMS & SOURCE \\
\hline American Samoa & 2003 & 1 & 35,709 & $\begin{array}{l}\text { National Agricultural Statistics Service - United States Department of Agriculture: National Agricultural } \\
\text { Census 2007. Online data: http://www.agcensus.usda.gov/Publications/2007/Full_Report/ }\end{array}$ \\
\hline Australia & 2007 & 6 & $94,199,025$ & $\begin{array}{l}\text { Australian Bureau of Statistics: Agricultural commodities: small area data, Australia 2006-2007 (GLIMS data } \\
\text { warehouse) }\end{array}$ \\
\hline Cook Islands & 2006 & 1 & 30,611 & Statistic Office: Census of Population Dwellings (2001 and 2006) (GLIMS data warehouse) \\
\hline New Caledonia & 2002 & 1 & 344,176 & $\begin{array}{l}\text { Institut de la Statistique et des Etudes Economiques (ISEE): Recensement Général Agricole (RGA) } 2002 . \\
\text { Online data: } \\
\text { http://www.davar.gouv.nc/portal/page/portal/davar/secteur_rural/statistiques_agricoles/donnees_synthe } \\
\text { se/recensement_agricole }\end{array}$ \\
\hline $\begin{array}{l}\text { Northern Mariana } \\
\text { Islands }\end{array}$ & 2007 & 1 & 11,812 & $\begin{array}{l}\text { National Agricultural Statistics Service - United States Department of Agriculture: National Agricultural } \\
\text { Census 2007. Online data: http://www.agcensus.usda.gov/Publications/2007/Full_Report/ }\end{array}$ \\
\hline Tonga & 2001 & 1 & 177,829 & $\begin{array}{l}\text { Ministry of Agriculture and Forestry, Kingdom of Tonga: Agriculture Census 2001. Online data: } \\
\text { http://www.spc.int/prism/tonga/index.php/surveys/agriculture-census-2011 }\end{array}$ \\
\hline
\end{tabular}

Guinea, Pitcairn, Samoa, Solomon Islands, Tokelau, Tuvalu, Vanuatu, Wake Island, Wallis and Futuna 
Supplementary information $5 \mathrm{~d}$ - Country details on sub-national statistics for ducks (year and level of observation) by continental tile.

Africa Tile (Data insufficient to run the regional model)

\begin{tabular}{|c|c|c|c|c|}
\hline COUNTRY & YEAR & LEVEL & Totals in GLIMS & SOURCE \\
\hline Burkina Faso & 2003 & 2 & 211,828 & $\begin{array}{l}\text { Ministère des Ressources Animales: Les Statistiques du Secteur de l'Élevage au Burkina Faso, } 2003 \text { \& } 2008 \\
\text { (GLIMS data warehouse) }\end{array}$ \\
\hline Cameroon & 1999 & 1 & 194,579 & $\begin{array}{l}\text { Direction des Etudes, de la Statistique et de la Coopération/MINEPIA (1999) et Institut National de la } \\
\text { Statistique (1998): Revue du sector avicole (GLIMS data warehouse) }\end{array}$ \\
\hline Congo & 2000 & 1 & 1,023 & $\begin{array}{l}\text { Ministère de l'Agriculture et de l'Élevage: Enquête Agricole Pilote, Campagne Agricole 1999-2000 (GLIMS } \\
\text { data warehouse) }\end{array}$ \\
\hline Egypt & 2011 & 1 & $4,658,920$ & $\begin{array}{l}\text { Central Agency for Public Mobilization and Statistics, Egypt: Estimated numbers of livestock and animals } \\
\text { by Governorate } 2011 \text { (GLIMS data warehouse) }\end{array}$ \\
\hline Ghana & 2009 & 1 & 330,045 & CountrySTAT - Food and agriculture data network (GLIMS data warehouse) \\
\hline Guinea & 2001 & 1 & 387,482 & $\begin{array}{l}\text { Service National des Statistiques Agricoles - Ministère de l'Agriculture, de l'Élevage et des Forêts: } \\
\text { Recensement national de l'agriculture, Campagne agricole 2000-2001 (GLIMS data warehouse) }\end{array}$ \\
\hline Guinea-Bissau & 1994 & 2 & 42,988 & $\begin{array}{l}\text { Instituto Nacional de Estadística: Inquérito Anual sobre Superficie Rendimento e Producao. Campanha } \\
\text { 93/94 (GLIMS data warehouse) }\end{array}$ \\
\hline Madagascar & 2005 & 2 & $24,213,521$ & $\begin{array}{l}\text { Ministère de l'Agriculture, Secrétariat General, Direction de la Prévision et des Etudes Economiques, } \\
\text { Madagascar: Recensement de l'agriculture (RA) - Campagne agricole 2004-2005 (GLIMS data warehouse) }\end{array}$ \\
\hline Malawi & 2007 & 2 & 429,170 & $\begin{array}{l}\text { Agriculture Statistics Division of the National Statistical Office of Malawi: National Census of Agriculture } \\
\text { and Livestock (October } 2006 \text { - October 2007) (GLIMS data warehouse) }\end{array}$ \\
\hline Mali & 2004 & 1 & 161,269 & $\begin{array}{l}\text { Ministère de l'Agriculture de l'Élevage et de la Pèche, République du Mali: Recensement de l'Agriculture } \\
\text { 2004-2005 (GLIMS data warehouse) }\end{array}$ \\
\hline Mozambique & 2010 & 1 & $1,881,736$ & National Directorate of Livestock: Censo AgroPecuario 2009-2010 (GLIMS data warehouse) \\
\hline Niger & 2007 & 1 & 427,747 & $\begin{array}{l}\text { Ministère des Ressources Animales: Recensement Général de l'Agriculture et du Cheptel (RGAC } \\
\text { 2005/2007). Online data: http://harvestchoice.org/publications/niger-recensement-general-de-lagriculture- } \\
\text { et-du-cheptel-rgac-20052007-volume-v-producti }\end{array}$ \\
\hline Nigeria & 2011 & 1 & $9,553,912$ & $\begin{array}{l}\text { National Bureau of Statistics, Federal Ministry of Agriculture and Rural Development: National Agriculture } \\
\text { Sample Survey 2010/2011 (GLIMS data warehouse) }\end{array}$ \\
\hline Rwanda & 2008 & 1 & 6,729 & Rwanda Animal Resources Development Authority (RARDA) (GLIMS data warehouse) \\
\hline $\begin{array}{l}\text { Sao Tome and } \\
\text { Principe }\end{array}$ & 1997 & 2 & 8,713 & $\begin{array}{l}\text { Ministère de l'Agriculture et de la Pêche: Enquête Agricole de Production (EAP) } 1997 \text { (GLIMS data } \\
\text { warehouse) }\end{array}$ \\
\hline
\end{tabular}


Sierra Leone

2004

2000

Uganda

United Republic of

2008

Tanzania
487,508 Ministry of Agriculture \& Food Security of Sierra Leone: 2004 Populations and Housing Census Statistical Office (GLIMS data warehouse)

13,200 Division de la Production et de la Santé Animales de la FAO, Centre d’Urgence pour les Maladies Animales Transfrontaliers, Unité de Socio-Économie, Production et Biodiversité. Revue du secteur avicole: Togo, 2008 (GLIMS data warehouse)

671,813 Uganda Bureau of Statistics: Uganda Census 2000 (GLIMS data warehouse)

1,156,726 Ministry of Water and Livestock Development of Tanzania: National Sample Census of Agriculture, 2007/2008 (GLIMS data warehouse)

No Data for: Algeria, Angola, Bassas da India, Benin, Botswana, British Indian Ocean Territory, Burundi, Côte d'Ivoire, Cape Verde, Central African Republic, Chad, Comoros, Democratic Republic of the Congo, Diibouti, Equatorial Guinea, Eritrea, Ethiopia, Europa Island, Gabon, Gambia, Glorioso Island, Juan de Nova Island, Kenya, Lesotho, Liberia, Libya, Mauritania, Mauritius, Mayotte, Morocco, Namibia, Réunion, Saint Helena, Senegal, Seychelles, Somalia, South Africa, Sudan, Swaziland, Tromelin Island, Tunisia, Western Sahara, Zambia, Zimbabwe 


\section{Asia Tile}

\begin{tabular}{|c|c|c|c|c|}
\hline COUNTRY & YEAR & LEVEL & Totals in GLIMS & SOURCE \\
\hline Afghanistan & 2003 & 2 & 387,038 & $\begin{array}{l}\text { Ministry of Agriculture, Irrigation and Livestock of Afghanistan: Afghanistan - National Livestock Census } \\
\text { 2002-2003 - Final Report. Online data: } \\
\text { http://reliefweb.int/sites/reliefweb.int/files/resources/58C374339FC582F149256DF30009D60F-faO-afg- } \\
\text { 4dec.pdf }\end{array}$ \\
\hline Bangladesh & 2005 & 6 & $37,932,995$ & $\begin{array}{l}\text { Bangladesh Bureau of Statistics: Data Retrieved } 12 \text { November } 2009 \text { from http://www.bbs.gov.bd/ } \\
\text { (GLIMS data warehouse) }\end{array}$ \\
\hline Belarus & 2005 & 1 & $1,017,938$ & $\begin{array}{l}\text { Ministry of Statistics and Analysis of The Republic of Belarus: Statistical Yearbook of the Republic of } \\
\text { Belarus } 2005 \text { (GLIMS data warehouse) }\end{array}$ \\
\hline Cambodia & 2009 & 2 & $5,122,207$ & $\begin{array}{l}\text { Department of Animal Production and Health, Cambodia: } 2009 \text { district data (David Bourn, } \\
\text { "Environmental Animal Health Management Initiative for Enhanced Smallholder Production", 2010) } \\
\text { (GLIMS data warehouse) }\end{array}$ \\
\hline China & 2005 & 6 & $931,845,945$ & $\begin{array}{l}\text { Editorial Department of China Animal Husbandry: China animal husbandry yearbook. Beijing, China: } \\
\text { China Agricultural Press (GLIMS data warehouse) }\end{array}$ \\
\hline India & 2007 & 2 & $27,154,998$ & $\begin{array}{l}\text { Department of Animal Husbandry, Dairying \& Fisheries, Ministry of Agriculture, India: Census of } \\
\text { Agriculture, } 2007 \text { (GLIMS data warehouse) }\end{array}$ \\
\hline Indonesia & 2010 & 1 & $44,301,804$ & Department Pertanian Republik Indonesia: Buku Statistik, 2011 (GLIMS data warehouse) \\
\hline $\begin{array}{l}\text { Lao People's } \\
\text { Democratic } \\
\text { Republic }\end{array}$ & 2009 & 2 & $6,279,320$ & $\begin{array}{l}\text { Department of Livestock and Fisheries, Laos: } 2009 \text { district data (David Bourn, “Environmental Animal } \\
\text { Health Management Initiative for Enhanced Smallholder Production", 2010) (GLIMS data warehouse) }\end{array}$ \\
\hline Malaysia & 2001 & 1 & $6,013,621$ & $\begin{array}{l}\text { Department of Veterinary Service, Malaysia: Livestock Products statistics 1996-2002 (GLIMS data } \\
\text { warehouse) }\end{array}$ \\
\hline Myanmar & 2006 & 6 & $9,745,621$ & $\begin{array}{l}\text { Livestock Breeding and Veterinary Department (LBVD), Ministry of Livestock Breeding and Fisheries, } \\
\text { Yangon, Myanmar: Myanmar Animal Census } 2006 \text { (GLIMS data warehouse) }\end{array}$ \\
\hline Nepal & 2000 & 3 & 425,158 & Ministry of Agriculture and Co-operatives, Agri-Business and Statistics Division (GLIMS data warehouse) \\
\hline Pakistan & 1996 & 2 & $1,127,108$ & $\begin{array}{l}\text { Agricultural Census Organization, Ministry of Food \& Agriculture, Pakistan: Livestock census } 1996 \text { - } \\
\text { special report. Lahore, Pakistan (GLIMS data warehouse) }\end{array}$ \\
\hline Philippines & 2006 & 3 & $7,739,879$ & $\begin{array}{l}\text { Bureau of Animal Industry, Philippines: } 2006 \text { data (David Bourn, “Environmental Animal Health } \\
\text { Management Initiative for Enhanced Smallholder Production”, 2010) (GLIMS data warehouse) }\end{array}$ \\
\hline Qatar & 2001 & 1 & 12,266 & $\begin{array}{l}\text { Ministry of Municipal Affairs and Agriculture Department of Agriculture and Water Research: Agricultural } \\
\text { Census 2000-2001 (GLIMS data warehouse) }\end{array}$ \\
\hline Republic of Korea & 2004 & 1 & $8,265,580$ & Korea National Statistical Office: Korea Statistical Yearbook 2005 (GLIMS data warehouse) \\
\hline
\end{tabular}


Romania

2002

Russian Federation

2006

2002

2004

2009

Turkey

Ukraine

Viet Nam

2009

2001

3,897,538 EUROSTAT on line database. Section: General Statistics, Region, Agriculture, Animal Populations and Land Use. February 2005. Online data:

http://epp.eurostat.ec.europa.eu/portal/page/portal/agriculture/data/

148,273 Russian Federation - Federal State Statistical Service: Results of All-Russia Agricultural Census 2006 (1-8 vol.) (GLIMS data warehouse)

23,940 Agriculture and Environment Statistics Division, Department of Census and Statistics, Sri Lanka: Census of Agriculture 2002, All Sectors. Online data: http://www.statistics.gov.lk/agriculture/AGC2002/AGC2002.htm

32,620,489 Department of Livestock Development, Ministry of Agriculture and Cooperatives, Thailand: Census 2004 (X- ray surveys searching for HPAI outbreaks) (GLIMS data warehouse)

413,523 Turkish Statistical Institute: Livestock statistics database 2009 (GLIMS data warehouse)

8,743,007 State Statistics Committee Ukraine: Head of livestock and poultry by regions (GLIMS data warehouse)

60,758,232 General Statistics Office of Vietnam: Agricultural Census database 2001. Online data: http://www.gso.gov.vn/default_en.aspx?tabid=477\&idmid=4\&ItemID=1824

No Data for: Armenia, Azerbaijan, Bahrain, Bhutan, Brunei Darussalam, Bulgaria, Christmas Island, Cocos (Keeling) Islands, Cyprus, Democratic People’s Republic of Korea, Georgia, Islamic

Republic of Iran, Iraq, Israel, Japan, Jordan, Kazakhstan, Kuril Islands, Kuwait, Kyrgyzstan, Lebanon, Liancourt Rock, Maldives, Mongolia, Oman, Paracel Islands, Republic of Moldova, Saudi Arabia,

Scarborough Reef, Senkaku Islands, Singapore, Spratly Islands, Syrian Arab Republic, Tajikistan, Timor-Leste, Turkmenistan, United Arab Emirates, Uzbekistan, Yemen 


\section{Europe Tile}

\begin{tabular}{|c|c|c|c|c|}
\hline COUNTRY & YEAR & LEVEL & Totals in GLIMS & SOURCE \\
\hline Albania & 2010 & 1 & 164,500 & Institute of Statics, Albania: Agricultural sector, Livestock 2001-2010 (GLIMS data warehouse) \\
\hline Austria & 2010 & 1 & $3,134,156$ & Statistics Austria: Livestock Survey, 2010 (GLIMS data warehouse) \\
\hline Belgium & 2010 & 2 & $6,429,600$ & $\begin{array}{l}\text { EUROSTAT online database. General Statistics, Region, Agriculture, Animal Populations and Land Use. } \\
\text { Online data: http://epp.eurostat.ec.europa.eu/portal/page/portal/agriculture/data/ }\end{array}$ \\
\hline $\begin{array}{l}\text { Bosnia and } \\
\text { Herzegovina }\end{array}$ & 2009 & 6 & 529,095 & $\begin{array}{l}\text { Federal Office of Statistics of Federation of Bosnia and Herzegovina: Number of animals from Federal } \\
\text { Agro-Mediterranean Institute of Mostar (GLIMS data warehouse) }\end{array}$ \\
\hline Croatia & 2003 & 1 & $1,726,895$ & $\begin{array}{l}\text { Central Bureau of Statistics, Republic of Croatia: Agricultural Census 2003. Online data: } \\
\text { http://www.dzs.hr/default_e.htm }\end{array}$ \\
\hline Czech Republic & 2010 & 2 & $1,906,929$ & $\begin{array}{l}\text { Czech Statistical Office: Agrocensus regions: Farm Structure Survey and Survey on Agricultural Production } \\
\text { Methods 2010. Online data: http://www.czso.cz/csu/2012edicniplan.nsf/engp/2127-12 }\end{array}$ \\
\hline Denmark & 2010 & 2 & $13,173,060$ & $\begin{array}{l}\text { Denmark Statistics: Farms by region and selected farms, crops and livestock. Online data: } \\
\text { http://www.statistikbanken.dk/statbank5a/SelectVarVal/Define.asp?Maintable=BDF51\&PLanguage=1 }\end{array}$ \\
\hline Estonia & 2001 & 2 & 327,195 & $\begin{array}{l}\text { Statistikaamet Statistical Office of Estonia: Agricultural Census 2001. Online data: } \\
\text { http://www.stat.ee/agricultural-census-2001 }\end{array}$ \\
\hline Finland & 2010 & 6 & $1,366,800$ & $\begin{array}{l}\text { The Information Centre of the Ministry of Agriculture and Forestry, Finland: Yearbook of Farm Statistics } \\
2011 \text { (GLIMS data warehouse) }\end{array}$ \\
\hline France & 2009 & 2 & $13,245,288$ & Service de la Statistique et de la Prospective (SSP): Statistique Agricole Annuelle (GLIMS data warehouse) \\
\hline Germany & 2007 & 2 & $25,247,881$ & $\begin{array}{l}\text { Federal Statistical Office, Germany: GENESIS database. Online data: } \\
\text { https://www.regionalstatistik.de/genesis/online/online;jsessionid=EE45147898822814978BE734145275C }\end{array}$ \\
\hline Greece & 2007 & 2 & $1,111,540$ & $\begin{array}{l}\text { 4?operation }=\text { sprachwechsel\&option }=\text { en } \\
\text { Hellenic Statistic Authority (EL.STAT): Farms and number of animals by type, region and county (GLIMS } \\
\text { data warehouse) }\end{array}$ \\
\hline Hungary & 2010 & 1 & $3,169,000$ & Hungarian Central Statistical Office: Regional Statistics. Online data: http://www.ksh.hu/agriculture \\
\hline Iceland & 2010 & 2 & 3,615 & Statistics Iceland: 2010 data (GLIMS data warehouse) \\
\hline Ireland & 2000 & 1 & $1,722,108$ & $\begin{array}{l}\text { Central Statistical Office Ireland: Census of Agriculture 2000. Online data: } \\
\text { http://www.cso.ie/en/releasesandpublications/agricultureandfishing/censusofagriculturemainresultsjune } 20 \\
\underline{00 /}\end{array}$ \\
\hline Italy & 2010 & 3 & $9,331,143$ & $\begin{array}{l}\text { Istituto Nazionale di Statistica: Censimento Agricoltura 2010. Online data: http://dati- } \\
\text { censimentoagricoltura.istat.it }\end{array}$ \\
\hline Latvia & 2001 & 1 & 368,909 & $\begin{array}{l}\text { Central Statistical Bureau of Latvia: Results of } 2001 \text { Agricultural Census. Online data: } \\
\text { http://www.csb.gov.lv/en/dati/agricultural-census-2001-30765.html }\end{array}$ \\
\hline
\end{tabular}




\begin{tabular}{|c|c|c|c|c|}
\hline Liechtenstein & 2009 & 6 & 1,811 & Amt für Statistik Liechtenstein: Landwirtschaftsstatistik 2009 (GLIMS data warehouse) \\
\hline Lithuania & 2010 & 2 & 942,163 & $\begin{array}{l}\text { Lietuvos Statistikas Departamenta: Number of livestock by administrative territory, } 2011 \text { (GLIMS data } \\
\text { warehouse) }\end{array}$ \\
\hline Luxembourg & 2008 & 2 & 77,841 & $\begin{array}{l}\text { Portail des statistiques du Grand-Duché de Luxembourg (STATEC): Recensement Agricole } 2008 \text { (GLIMS } \\
\text { data warehouse) }\end{array}$ \\
\hline Malta & 2010 & 6 & 70,593 & $\begin{array}{l}\text { National Statistics Office of Malta: Census of Agriculture 2010. Online data: } \\
\text { http://www.nso.gov.mt/statdoc/document_view.aspx?id=3287\&backurl=/themes/theme_page.aspx }\end{array}$ \\
\hline Montenegro & 2010 & 1 & 41,118 & $\begin{array}{l}\text { Statistical office of Montenegro (MONSTAT): Agriculture Census 2010. Online data: } \\
\underline{\text { http://www.monstat.org/eng/page.php?id=58\&pageid }=58}\end{array}$ \\
\hline Netherlands & 2010 & 2 & $12,254,972$ & $\begin{array}{l}\text { Centraal Bureau voor de Statistiek, Netherlands: Agriculture: crops, livestock and land use by general far } \\
\text { type, region - } 2011 \text { (GLIMS data warehouse) }\end{array}$ \\
\hline Norway & 2010 & 1 & $1,607,772$ & Statistics Norway: Agriculture Statistics 2011 (GLIMS data warehouse) \\
\hline Poland & 2009 & 1 & $14,278,600$ & Central Statistical Office, Poland: Statistical Yearbook of Agriculture 2010 (GLIMS data warehouse) \\
\hline Portugal & 2009 & 2 & $1,851,290$ & $\begin{array}{l}\text { Instituto Nacional de Estatística - Statistics Portugal: Agricultural Census 2009. Online data: } \\
\underline{\text { http://ra09.ine.pt/xportal/xmain?xpid=RA2009\&xpgid=ra_home }}\end{array}$ \\
\hline Serbia & 2006 & 1 & $3,998,927$ & Statistical Office of Serbia: Municipalities of Serbia 2007 (GLIMS data warehouse) \\
\hline Slovakia & 2009 & 2 & 740,862 & $\begin{array}{l}\text { Statistical Office of the Slovak Republic: Livestock by territory, products and period. Online data: } \\
\text { http://px-web.statistics.sk/PXWebSlovak/DATABASE/En/databasetree.asp }\end{array}$ \\
\hline Slovenia & 2010 & 2 & 308,540 & $\begin{array}{l}\text { Statistical Office of the Republic of Slovenia: Agricultural Census Slovenia 2010. Online data: } \\
\text { http://www.stat.si/eng/novica_prikazi.aspx?id=4594 }\end{array}$ \\
\hline Spain & 2010 & 2 & $25,704,041$ & $\begin{array}{l}\text { Ministerio de Medio Ambiente y Medio Rural y Marino, Gobierno de España (MARM): Encuestas } \\
\text { Ganaderas, } 2010 \text { (GLIMS data warehouse) }\end{array}$ \\
\hline Sweden & 2009 & 1 & $1,528,740$ & Statistics Sweden: Yearbook of Agricultural Statistics 2010 (GLIMS data warehouse) \\
\hline Switzerland & 2009 & 1 & $1,557,204$ & Swiss Federal Statistical Office: Recensement des entreprises agricoles,1999-2009 (GLIMS data warehouse) \\
\hline $\begin{array}{l}\text { The former } \\
\text { Yugoslav Republic } \\
\text { of Macedonia }\end{array}$ & 2006 & 2 & 173,185 & $\begin{array}{l}\text { State Statistic Office of the Republic of Macedonia: Census of Agriculture 2007. Online data: } \\
\text { http://www.stat.gov.mk/PrikaziPublikacija_en.aspx?id=51\&rbr=205 }\end{array}$ \\
\hline United Kingdom & 2009 & 2 & $4,525,710$ & $\begin{array}{l}\text { Office for National Statistics, U.K.: Statistics by Region. Online data: } \\
\text { http://www.statistics.gov.uk/hub/regional-statistics/ }\end{array}$ \\
\hline
\end{tabular}

No Data for: Andorra, Faroe Islands, Gibraltar, Guernsey, Holy See, Isle of Man, Jersey, Madeira Islands, Monaco, San Marino, Svalbard and Jan Mayen Islands 


\section{North America Tile}

\begin{tabular}{|c|c|c|c|c|}
\hline COUNTRY & YEAR & LEVEL & Totals in GLIMS & SOURCE \\
\hline Belize & 2003 & 1 & 21,224 & $\begin{array}{l}\text { Ministry Of Agriculture, Fisheries and Cooperatives, Belize: Annual Report 2003. Online data: } \\
\text { http://www.agriculture.gov.bz/PDF/Annual_Report_2003.pdf }\end{array}$ \\
\hline Canada & 2006 & 2 & $14,168,313$ & $\begin{array}{l}\text { Statistics Canada: } 2006 \text { Census of Agriculture. Online data: http://www.statcan.gc.ca/ca-ra2006/index- } \\
\text { eng.htm }\end{array}$ \\
\hline Cayman Islands & 2009 & 1 & 1,063 & $\begin{array}{l}\text { Economics and Statistics Office - Government of the Cayman Islands: Statistical Compendium } 2009 \\
\text { (GLIMS data warehouse) }\end{array}$ \\
\hline Costa Rica & 2006 & 1 & 297,359 & $\begin{array}{l}\text { Ministerio de Agricultura y Ganadería, Costa Rica: Programa Nacional de Sanidad Porcina } 2006 \text { (GLIMS } \\
\text { data warehouse) }\end{array}$ \\
\hline El Salvador & 2007 & 2 & 165,866 & Ministerio de Economía - El Salvador: IV Censo Agropecuario 2007-2008 (GLIMS data warehouse) \\
\hline Guadeloupe & 2000 & 1 & 25,839 & $\begin{array}{l}\text { Agreste la Statistique Agricole, Le Ministère de l'Agriculture, de l'Alimentation, de la Pêche et de la Ruralité, } \\
\text { France: Recensement Agricole 2000. Online data: http://agreste.agriculture.gouv.fr/recensement-agricole- } \\
\text { 2010/resultats-donnees-chiffrees/ }\end{array}$ \\
\hline Guatemala & 2003 & 2 & 419,170 & $\begin{array}{l}\text { Instituto Nacional de Estadística, Guatemala: IV Censo Nacional Agropecuario 2003. Online data: } \\
\text { http://www.ine.gob.gt/np/agropecuario/tomo\%20IV.pdf }\end{array}$ \\
\hline Jamaica & 2009 & 1 & 361,540 & $\begin{array}{l}\text { Agricultural Business Information System, Jamaica: Livestock Summary Report, } 2009 \text { (GLIMS data } \\
\text { warehouse) }\end{array}$ \\
\hline Martinique & 2000 & 1 & 20,621 & $\begin{array}{l}\text { Agreste la Statistique Agricole, Le Ministère de l'Agriculture, de l'Alimentation, de la Pêche et de la Ruralité, } \\
\text { France: Recensement Agricole 2000. Online data: http://agreste.agriculture.gouv.fr/recensement-agricole- } \\
\text { 2010/resultats-donnees-chiffrees/ }\end{array}$ \\
\hline Mexico & 2007 & 2 & $8,964,101$ & $\begin{array}{l}\text { Instituto Nacional de Estadística Geografía e Informática, México: Censo Agrícola, Ganadero y Forestal } \\
\text { 2007. Online data: } \\
\text { http://www.inegi.org.mx/est/contenidos/proyectos/Agro/ca2007/Resultados_Agricola/default.aspx }\end{array}$ \\
\hline Nicaragua & 2001 & 2 & 383,172 & $\begin{array}{l}\text { Instituto Nacional de Estadística y Censos, Nicaragua: III Censo Nacional Agropecuario, } 2001 \text { (GLIMS } \\
\text { data warehouse) }\end{array}$ \\
\hline Panama & 2001 & 2 & 312,102 & $\begin{array}{l}\text { Instituto Nacional de Estadística y Censo: Existencia de animales en la República, por clase de animal, } \\
\text { según provincia: } 21 \text { de abril de } 1991 \text { al } 22 \text { abril de 2001. Online data: } \\
\text { http://www.contraloria.gob.pa/inec/Avance/Avance.aspx?ID_CATEGORIA=2\&ID_CIFRAS=8\&ID_I } \\
\text { DIOMA=1 }\end{array}$ \\
\hline Puerto Rico & 2007 & 2 & 63,585 & $\begin{array}{l}\text { National Agricultural Statistics Service - United States Department of Agriculture: National Agricultural } \\
\text { Census 2007. Online data: http://www.agcensus.usda.gov/Publications/2007/Full_Report/ }\end{array}$ \\
\hline
\end{tabular}




\section{Saint Lucia}

Saint Vincent and

the Grenadines

United States of$$
2000
$$

America Census 2007. Online data: http://www.agcensus.usda.gov/Publications/2007/Full_Report/

19,520 Ministry of Agriculture, Forestry and Fisheries: Census of Agriculture - Final report 2007. Online data: http://www.govt.lc/www/publications/AgricultureCensus2007.pdf

4,807 Ministry of Agriculture and Labour, Government of Saint Vincent and the Grenadines: National Agricultural Census 2000 (GLIMS data warehouse)

Netherlands Antilles, Saint Kitts and Nevis, Turks and Caicos Islands, United States Virgin Islands 
South America Tile (Data insufficient to run the regional model)

\begin{tabular}{|c|c|c|c|c|}
\hline COUNTRY & YEAR & LEVEL & Totals in GLIMS & SOURCE \\
\hline Colombia & 2001 & 1 & 546,272 & $\begin{array}{l}\text { Ministerio de Agricultura y Desarrollo Rural, Departamento Administrativo Nacional de Estadística, } \\
\text { Colombia: Encuesta Nacional Agropecuaria - Resultados } 2001 \text { (GLIMS data warehouse) }\end{array}$ \\
\hline Ecuador & 2000 & 2 & 351,945 & $\begin{array}{l}\text { Project SICA, Agricultural Information System, Ministry of Agriculture and Livestock, Ecuador: III Censo } \\
\text { Nacional Agropecuario (GLIMS data warehouse) }\end{array}$ \\
\hline Paraguay & 2001 & 1 & 717,600 & Dirección General de Estadística, Encuestas y Censos: Anuario Estadístico 2001 (GLIMS data warehouse) \\
\hline $\begin{array}{l}\text { Venezuela, } \\
\text { Bolivarian Republic }\end{array}$ & 2007 & 1 & 142,724 & $\begin{array}{l}\text { Ministerio del Poder Popular para la Agricultura y Tierras - Venezuela: VII Censo Agrícola 2007. Online } \\
\text { data: http://censo.mat.gob.ve/ }\end{array}$ \\
\hline
\end{tabular}




\section{Oceania Tile}

\begin{tabular}{|c|c|c|c|c|}
\hline COUNTRY & YEAR & LEVEL & Totals in GLIMS & SOURCE \\
\hline Australia & 2007 & 6 & $3,042,342$ & $\begin{array}{l}\text { Australian Bureau of Statistics: Agricultural commodities: small area data, Australia 2006-2007 (GLIMS data } \\
\text { warehouse) }\end{array}$ \\
\hline Cook Islands & 2006 & 1 & 436 & Statistic Office: Census of Population Dwellings (2001 and 2006) (GLIMS data warehouse) \\
\hline Fiji & 2009 & 2 & 72,486 & $\begin{array}{l}\text { Department of Agriculture Economic Planning and Statistic Division: National Agricultural Census } 2009 \\
\text { report. Online data : } \\
\text { http://www.fao.org/fileadmin/templates/ess/ess_test_folder/World_Census_Agriculture/Country_info__ } \\
\text { 2010/Reports/Reports_3/FJI_ENG_REP_2009.pdf }\end{array}$ \\
\hline New Caledonia & 2002 & 2 & 9,844 & $\begin{array}{l}\text { Institut de la Statistique et des Etudes Economiques (ISEE): Recensement Général Agricole (RGA) } 2002 . \\
\text { Online data: } \\
\text { http://www.davar.gouv.nc/portal/page/portal/davar/secteur_rural/statistiques_agricoles/donnees_synthe } \\
\text { se/recensement_agricole }\end{array}$ \\
\hline $\begin{array}{l}\text { Northern Mariana } \\
\text { Islands }\end{array}$ & 2007 & 1 & 189 & $\begin{array}{l}\text { National Agricultural Statistics Service - United States Department of Agriculture: National Agricultural } \\
\text { Census 2007. Online data: http://www.agcensus.usda.gov/Publications/2007/Full_Report/ }\end{array}$ \\
\hline Tonga & 2001 & 1 & 1,119 & $\begin{array}{l}\text { Ministry of Agriculture and Forestry, Kingdom of Tonga: Agriculture Census 2001. Online data: } \\
\text { http://www.spc.int/prism/tonga/index.php/surveys/agriculture-census-2011 }\end{array}$ \\
\hline
\end{tabular}

